\title{
A multi-layered fast marching method for unmanned surface vehicle path planning in a time-variant maritime environment
}

\author{
Rui Song, Yuanchang Liu ${ }^{1}$ and Richard Bucknall \\ Department of Mechanical Engineering, University College London, Torrington
}

Place, London WCIE 7JE, UK

\begin{abstract}
Concerns regarding the influence of the marine environment, such as surface currents and winds, on autonomous marine vehicles have been raised in recent years. A number of researchers have been working on the development of intelligent path planning algorithms to minimise the negative effects of environmental influences, however most of this work focuses on the platform of autonomous underwater vehicles (AUVs) with very little work on unmanned surface vehicles (USVs). This paper presents a novel multi-layered fast marching (MFM) method developed to generate practical trajectories for USVs when operating in a dynamic environment. This method constructs a synthetic environment framework, which incorporates the information of planning space and surface currents. In terms of the planning space, there are repelling and attracting forces, which are evaluated using an attractive/repulsive vector field construction process. The influence of surface currents is weighted against the

1 Corresponding author. Tel.: +44(0)20 31089495.

E-mail address: yuanchang.liu.10@ucl.ac.uk (Y. Liu).
\end{abstract}


obstacles in the planning space using a 4-regime risk strategy. A trajectory is then calculated using the anisotropic fast marching method. The complete algorithm has been tested and validated using simulated surface currents, and the performance of generated trajectories have been evaluated in terms of different optimisation criteria, such as the distance and energy consumption.

Key words: Anisotropic fast marching; Marine environment; Path planning; Surface currents; Unmanned surface vehicle 


\section{Nomenclature}

Roman symbols

$A \quad$ A matrix that determines the strength of the simulated currents data

AR Anisotropic ratio

$C \quad$ A constant equalling to $\rho / c$

$c$ the nominal speed of the USV

$C_{\text {free }} \quad$ Collision-free space

$C_{o b s} \quad$ Obstacle space

$C$-space Configuration space

$D_{\text {att }}, D_{\text {rep }} \quad$ Attractive and repulsive potential fields

$D_{\text {cum }} \quad$ The total distance cost

$d_{i} \quad$ The length of $i$ th path segment

$d_{S A} \quad$ The minimum distance that the USV should keep away from the obstacle

$e_{\vec{p}} \quad$ The unit vector of USV heading at $\vec{p}$

$\boldsymbol{F}_{\text {att }}, \boldsymbol{F}_{\text {rep }} \quad$ Attractive and repulsive vector fields

$\boldsymbol{F}_{\text {base }} \quad$ Base layer vector field

$\boldsymbol{F}_{\text {env }} \quad$ Environment layer vector field

$\boldsymbol{F}_{s y n} \quad$ Synthetic vector field

$h \quad$ step size

$J \quad$ The integration of the relative velocity of USV to the surface currents

$\vec{M} \quad$ Intersection point of the optimal path and $\vec{p}_{i} \vec{p}_{j}$

min_Obs The minimum distance to the obstacles

$\vec{n} \quad$ Local ellipse direction

$\vec{p} \quad$ Grid point position

$\vec{p}_{i}, \vec{p}_{j} \quad$ Neighbouring points of $\vec{p}$

$\vec{p}_{\text {obs }} \quad$ Obstacles' locations

$\vec{p}_{\text {start }}, \vec{p}_{\text {goal }} \quad$ Positions of start and goal points

$r \quad$ Wave propagation speed along $\vec{\theta}$

$R_{\beta_{1}, \beta_{2}} \quad$ Risk regime

$r_{a}, r_{b} \quad$ Major and minor radii along the $X$ and $Y$ axes of the local ellipse frame

$\vec{r}_{a}, \vec{r}_{b} \quad$ The major and minor axes of the local ellipse frame

$\Delta t \quad$ Time step

$U(\vec{p}) \quad$ Wave arrival time at $\vec{p}$

$u_{\vec{p}_{i} \vec{p}_{j}}(\vec{p}) \quad$ Temporary cost at $\vec{p}$

$\boldsymbol{V}_{u}, \boldsymbol{V}_{v} \quad$ Two orthogonal components of currents vector field 
$W$

The total energy cost

$W_{i} \quad$ The power consumption at each position

Greek symbols

$\alpha \quad$ Propagation scale limit

$\beta_{1}, \beta_{2} \quad$ Field weightings of $\boldsymbol{F}_{\text {env }}$ and $\boldsymbol{F}_{\text {base }}$ respectively

$\varphi \quad$ Angle between $\vec{n}$ and $X$ axis

$\rho \quad$ The water density

$\tau(\vec{p}) \quad$ Wave propagation speed related to $\vec{p}$

$\tau(\vec{\theta}(t)) \quad$ Wave propagation speed related to $\vec{p}$ and orientation

$\vec{\theta}(t) \quad$ Cost/speed vector 


\section{Introduction}

Unmanned surface vehicles (USVs) can be used in various marine applications. When operating individually, USVs can be deployed in pollutant tracking missions (Xu et al. [2006]) as well as environmental and hydrographic surveys (Caccia et al. [2005]). In addition, when collaborating with autonomous underwater vehicles (AUVs), a USV can be used as the mother ship to monitor a mission (Alves et al. [2006]) and as a platform for the launch and recovery of AUVs (Ferreira et al. [2006]). To successfully complete such missions, it is necessary to improve reliability and autonomy of the USV.

Path planning is a critical part in the USV's development, with the aim of using the algorithm to determine the optimal trajectory to guide the USV's voyage. It not only determines the level of autonomy of the vehicle, but it is also the premise of the reliability of a mission and the likelihood of success (Statheros et al. [2008]). When developing the algorithm, factors such as the total path distance as well as safety are main concerns (LaValle [2006]). In addition, the quality of the generated trajectory, such as smoothness and continuity, also needs to be taken into account (Smierzchalski [1999]). Path planning algorithms can be generally divided into two categories: the pregenerative approach (path generated prior to launching the USV), such as Chen et al. [1995], and the reactive approach (path generated while the vehicle is en route), such as Kamon and Rivlin [1995], which is regarded as the 'dynamic path planning approach'. To calculate the path, different computational methods can be applied such as genetic algorithms (GAs), graph search techniques and artificial potential field 
methods amongst others.

GAs generate a population of possible paths which are evolved iteratively, using genetic operators (such as the mutation and crossover) (Goldberg [1989]) to pursue optimal results. However, drawbacks to GAs include a lack of convergence, which means the generated path may be suboptimal, as well as a lack of consistency, which makes the vehicle's trajectories difficult to track.

Compared with GAs, graph search techniques such as A* and Dijkstra's methods have better consistency and convergence because they use a discretised representation of the environment, known as a grid map. However, as a result of the non-holonomic constraint of the vehicles, a further path smoothing procedure is needed (Petres et al. [2007]). Moreover, the computational time can be potentially high. The computational time is proportional to the number of grid points on the map, which is in turn dependent on the resolution of the graph (finer or coarser). Rapidly exploring random tree (RRT) approaches introduced by LaValle [1998] do not need to explicitly set any resolution parameters so the RRT method has the ability to explore the environment space quickly and uniformly using a random sampling scheme. However, the RRT approach is not suitable in the scenario of dynamic path planning as they are incapable of providing a global optimal solution with the least distance cost (Lolla et al. [2014]).

Potential field algorithms search the path by constructing an artificial potential field (APF) to weigh the influences of obstacles and goal points (Andrews [1983] and Khatib 
[1986]). These algorithms are computationally efficient, but are susceptible to the local minima problem (the vehicle can be trapped in a U-shaped obstacle) (Andrews [1983]). To address the defect of these algorithms, Wu et al. [2015] have proposed a modified APF method to improve the performance of path planning. The local minima problem has been addressed by integrating a wall-following method, which enables the vehicle to move away from the 'trapped' point by following the edge of the obstacle. In addition, a combinatorial strategy has been proposed by combining the APF with the ant colony optimisation (ACO). The ACO is utilised for global path planning with the generated path being used as the primary guidance route. When the vehicle encounters a moving obstacle, or experiences a change of the environment, the APF will be used as a local path planner to modify the path and avoid collisions. However, such an algorithm may increase the computational burden because additional algorithms are added. An alternative is to create the potential field which has no local minima. Garrido et al. [2008] therefore applies the fast marching (FM) method to construct such a field by simulating electromagnet wave propagation. The wave starts from the mission start point and continues to iterate until reaching the end point. The generated field will only have one global minima point which is located at the start point with the potential value being 0 .

It should be noted that the majority of the aforementioned studies focus on generating a collision free path but ignore environmental impact on the vehicles. The marine environment is an uncertain, complex and volatile space which impacts path planning 
as evidenced from experiments carried out by Song [2014] when a discrepancy caused by surface currents was found to exist between a planned path and the actual trajectory track taken by a USV. Such discrepancy can jeopardise marine vehicles' missions, especially when vehicles have limited operating speed and relatively small dimensions and displacements. It is therefore very important to consider the influences of environmental factors when developing the path planning algorithms for marine vehicles.

Agarwal and Lermusiaux [2011] used the level set method to solve the environmental influence problem for AUV path planning. Petres et al. [2005] used the anisotropic fast marching (AFM) method to address similar problems but in an environment where relatively stronger currents exist. The AFM is an improved version of the FM method with higher computational efficiency than the level set method (Agarwal and Lermusiaux [2011]). Also, the optimal collision free path generated by the AFM is able to provide the guaranteed convergence, which has been intuitively explained in Konukoglu et al. [2007] and mathematically proven in Mirebeau [2014]. However, these studies have only been applied on AUV platforms, where only the constraints of deep ocean currents and collision avoidance (limited distance to the obstacles) are considered. For surface vehicle navigation, additional constraints such as wind, tidal currents and traffic regulations such as COLREGs also need to be considered, for which the conventional AFM cannot implement.

To address the shortcomings, an improved AFM named as the multi layered fast 
marching (MFM) method has been proposed with initial work presented in Song et al. [2015]. However, the improved AFM only considers a time-invariant environment with no surface current changes. Additionally, an obstacle's impact on the USV is assumed to be uniform regardless of location changes. In this paper the framework has been improved by adding a geometrical analysis to assist with minimising the negative effects from both physical obstacles (coastal lines and islands) and environmental factors, such as currents and wind. An attractive/repulsive vector field construction process, a 4-regime risk strategy and two operation handlers have been developed to process and evaluate the environmental conditions. These modifications make important improvements to the method with the main focus being on generating a feasible trajectory in the presence of dynamic surface currents, while striving for distance and energy optimisation. This new multi-layered concept can facilitate the path planning algorithm to generate an optimal trajectory by layering various constraint costs in a complex environment.

This paper is organised as follows: Section 2 defines the problem and introduces the relevant notation. Section 3 briefly explains the conventional FM and the AFM methods, which have been adopted as the basis of the proposed algorithm. The structure and implementation of the new path planning algorithm are discussed in Section 4. Simulation results in self-constructed environments and complex environments are presented in Section 5. Conclusions are presented at Section 6. 


\section{Problem statement}

To achieve the autonomous navigation of USVs in the practical maritime environment, two critical problems need to be addressed. The first is: Can the algorithm intelligently prioritise a number of different missions in different environments? For example, when the USVs take small-scale ocean missions, such as the bathymetric surveys and data collection in hostile areas or cluttered environments, the collision avoidance capability has the highest priority compared to other requirements. Comparatively, undertaking large-scale missions, such as cooperating with AUVs to observe ocean movements, energy efficiency becomes critical. Interest now lies in increasing the endurance of the USV, hence an energy efficient path planning algorithm is necessary.

The second problem is: Can the algorithm generate a feasible and optimal trajectory in a complex maritime environment including changing surface currents? Surface currents have a direct effect on the displacement of the vehicle along its generated trajectory. Especially in strong surface currents, how best to make the trade off between the optimisation criteria, such as safety and other costs including energy cost, travel time, and trajectory distance? Downstream surface currents can help to reduce energy consumption, but it may sacrifice the distance cost or even total energy cost hence, the question is complex to answer. In some circumstances, such as unflavoured surface currents, cross currents operation is necessary. 
To address the aforementioned problems and challenges, a hybrid algorithm combining the conventional algorithms becomes necessary in order to optimise the path planning of a USV. Whilst the potential field method potentially offers a good solution the challenges are:

- Develop a pre-analysis process to construct a comprehensive environment framework representing the environment of interest.

- Design and employ the governing principles and equations to evaluate the obstacles and environmental influence priorities.

- Apply the path searching algorithm to generate an optimal trajectory.

\subsection{Environment framework}

In the majority of previous work, the path planning algorithm has been primarily applied on the configuration space (C-space), which consists of obstacle space $\left(C_{o b s}\right)$ and collision-free space $\left(C_{\text {free }}\right)$. Within the $C$-space, the movement of the USV can be represented as a configuration, i.e. a vector specifying all the motions of the USV such as its position and orientation (LaValle [2006]). Overall, $C$-space is a set of all possible configurations.

A new environment framework is constructed here to represent both obstacles and environmental influence in the form of a vector field. This framework is called the Synthetic Vector Field $\left(\boldsymbol{F}_{\text {syn }}\right)$ and includes two main layers: 
1) The $C$-space is the base layer of the $\boldsymbol{F}_{\text {syn }}$, denoted as $\boldsymbol{F}_{\text {base, }}$, which can be obtained from the data acquisition module of the USV's navigation system (Liu et al [2015]). Normally, the base layer is represented as a binary grid map, where the obstacle and collision free space are distinguished in black and white. Configuration at each grid has its coordinate relative to the $C$-space frame coordinate, and has the corresponding weighting with values of $0\left(C_{o b s}\right)$ or $1\left(C_{\text {free }}\right)$. Only static obstacles, such as islands and coastlines are considered.

2) The environmental influence factors such as surface currents are represented as the environment layer, denoted as $\boldsymbol{F}_{\text {env }}$. Here, it is assumed that the surface currents are known in advance. Such data are available from a tidal atlas or admiralty charts for different time periods and are predictable for different astronomical influences. Even though the data associated with surface currents contain uncertainties, it is a reasonable assumption that satisfactory currents data models can be established by calculating the mode or the mean of the predicted surface currents. In addition, it was assumed in Lolla et al. [2015] that the distance travelled by a USV is much longer than the vehicle's overall length, hence the interaction between the vehicle and the currents can be regarded as purely kinematic, namely the fluid dynamics are ignored.

As the $\boldsymbol{F}_{\text {env }}$ is represented in the vector field format, the $\boldsymbol{F}_{\text {base }}$ needs to be vectorised to have the same format, which can be achieved using the conventional FM method and 
the central differential method. The details of the vectorisation and the construction of $\boldsymbol{F}_{\text {base }}$ will be explained in Sections 4.1 and 4.2, respectively. 


\section{Methodologies}

The conventional FM method and the AFM method are the main methodologies used to create the comprehensive environment framework $\left(\boldsymbol{F}_{s y n}\right)$ and to calculate an optimal trajectory.

\subsection{Fast marching method}

The FM method is a level-set method introduced in imaging processing (Caselles et al. [1995]). Its aim is to generate an arrival time map $U$ that satisfies the Eikonal equation, which describes a wave front propagation scenario. The Eikonal equation can be expressed in the form of:

$$
\|\nabla U(\vec{p})\| \tau(\vec{p})=1
$$

where $\tau(\vec{p})$ is the wave propagation speed that is related to position $\vec{p}=(x, y)$. The solution $U(\vec{p})$ is the wave arrival time at $\vec{p}$. Note that $U(\vec{p})$ can be interpreted as the distance cost from the start point to $\vec{p}$, if the wave propagation has a constant speed.

Both Sethian [1996] and Tsitsiklis [1995] have provided an individual algorithm to numerically solve the Equation (1). Tsitsiklis's method will be explained in detail as its concept has been adopted as the basis for the AFM method to search for the optimal path in $\boldsymbol{F}_{\text {syn. }}$. 
As shown in Fig. 1(a), if the optimal path to $\vec{p}$ arrives from northwest and intersects $\vec{p}_{i} \vec{p}_{j}$ at $\vec{M}$, the arrival time at $\vec{p}$ can be computed from $\vec{p}_{i}$ and $\vec{p}_{j}$, denoted as $u_{\vec{p}_{i}} \vec{p}_{j}(\vec{p})$, and expressed as:

$$
u_{\vec{p}_{i} \vec{p}_{j}}(\vec{p})=\min _{t_{1} t_{2}}\left(t_{1} u_{\vec{p}_{i}}+t_{2} u_{\vec{p}_{j}}+\frac{\sqrt{t_{1}^{2}+t_{2}^{2}}}{\tau(\vec{p})}\right)
$$

where $u_{\vec{p}_{i}}$ and $u_{\vec{p}_{j}}$ are the arrival time at $\vec{p}_{i}$ and $\vec{p}_{j}$ respectively. $t_{1}$ and $t_{2}$ satisfy the following conditions: $t_{1}+t_{2}=1$ and $t_{1}, t_{2}>0$. Note that $U(\vec{p})$ in Equation (1) represents the total cost at the grid point $\vec{p}$. The value of $U(\vec{p})$ is fixed and does not change while the algorithm is iterating; whereas, $u_{\vec{p}_{\vec{p}} \vec{p}_{j}}(\vec{p})$ in Equation (2) is a temporary cost at $\vec{p}$, which is calculated from neighbouring $\vec{p}_{i}$ and $\vec{p}_{j}$. In this paper, the 4-geometry connection scheme ( 8 neighbours around $\vec{p}$, see Fig. $1 \mathrm{~b}$ ) is adopted to describe the neighbour points allocation. Therefore, eight different $u_{\vec{p}_{i}} \vec{p}_{j}(\vec{p})$ values will be calculated and compared. The smallest one will be selected and set as $U(\vec{p})$.

As a grid map based algorithm, the time complexity of the FM is $O(N \log N)$, where $N$ is the total number of grid points in the domain of interest. In addition, the travel of the wave front follows a circular cost/speed characteristic at each $\vec{p}$ so there is no directional preference, which means that the 8 neighbouring points of $\vec{p}$ have the same priority when they are updated.

\subsection{Anisotropic fast marching method}

It is required to take the effect of vector orientation into consideration when the path planning algorithm is applied to the synthetic vector field $\left(\boldsymbol{F}_{\text {syn }}\right)$, where any useful 
information is expressed in the vector form. However, from Equation (2), it can be observed that the conventional FM method only takes the distance cost into account. To integrate the orientation information, the conventional FM method was improved in Lin [2003], to a new algorithm named as the 'anisotropic fast marching algorithm' (AFM). The AFM algorithm uses a similar strategy to the FM method making the AFM's time complexity remain as $O(N \log N)$, where $N$ is the total number of grid points in the domain of interest. The AMF algorithm is described in Algorithm 1. There are three types of point sets defined as:

- the Accepted set in Algorithm 1 is defined as the set of the configuration points with the updated $U$ values. The $U$ values will not be changed when the algorithm is executing;

- the Trial set is the set of configuration points that have had their $U$ values calculated and that are going to be updated;

- and the Far set is the set of all other configuration points that have not had their $U$ values computed.

The updating scheme of the AFM is described in Algorithm 2, where $u_{\vec{p}_{i}} \vec{p}_{j}(\vec{p})$ is a temporary $U$ value of $\vec{p}$ and is expressed as,

$$
u_{\vec{p}_{i} \vec{p}_{j}}(\vec{p})=\min _{t \in[0,1]}\left(t u_{\vec{p}_{i}}+(1-t) u_{\vec{p}_{j}}+\frac{\|\vec{\theta}(t)\|}{\tau(\vec{\theta}(t))}\right)
$$

Here $\vec{\theta}(t)=\vec{p}-\left(t \vec{p}_{i}+(1-t) \vec{p}_{j}\right)$ is a vector that gives the direction of the cost/speed profile (see in Fig. $2 \mathrm{~b}$ ). $\|\vec{\theta}(t)\|$ is the distance between $\vec{p}$ and the intersection point 
between $\vec{p}_{i}$ and $\vec{p}_{j}$. The wave propagation speed is now dependent on orientation as denoted as $\tau(\overrightarrow{\theta(}(t))$. To simplify the notation, $\vec{\theta}(t)$ is replaced as $\vec{\theta}$ in the following sections.

In contrast to the conventional FM method, the local cost/speed characteristic of the AFM method is no longer circular. In Lin [2003], an elliptical shape was used to represent the local cost/speed model as it is easy to convert to a circle (conventional FM case). The direction of each vector on the synthetically generated vector field is defined as the major axis of the ellipse; while its minor axis is perpendicular to the vector's direction. In this case, the wave front travels along the major axis as the propagation preferred direction. In the general case, the ellipse speed profile is described as:

$$
\frac{x^{2}}{r_{a}^{2}}+\frac{y^{2}}{r_{b}^{2}}=1
$$

where $r_{a}$ and $r_{b}$ are the major and minor radii along the $X$ and $Y$ axes of the local ellipse frame. If the ellipse is along a direction of $\vec{n}$, and the wave front comes from the first octant (as shown in Fig. 2b), then the radius $r$ along $\vec{\theta}$, satisfies:

$$
r^{2}\left(\frac{(\cos \angle(\vec{\theta}, \vec{n}))^{2}}{r_{a}^{2}}+\frac{(\sin \angle(\vec{\theta}, \vec{n}))^{2}}{r_{b}^{2}}\right)=1
$$

where $\angle(\vec{\theta}, \vec{n})$ is the angle between $\vec{\theta}$ and $\vec{n}, r$ is used as the wave propagation speed along the $\vec{\theta}$ direction, hence $\tau(\overrightarrow{\theta)}$ in Equation (3) can now be written as:

$$
\tau(\vec{\theta})=\frac{1}{\sqrt{\frac{(\cos \angle(\vec{\theta}, \vec{n}))^{2}}{r_{a}^{2}}+\frac{(\sin \angle(\vec{\theta}, \vec{n}))^{2}}{r_{b}^{2}}}}=\frac{\|\vec{\theta}\|}{\sqrt{\frac{\left(\theta_{x} \cos \varphi\right)^{2}}{r_{a}^{2}}+\frac{\left(\theta_{y} \sin \varphi\right)^{2}}{r_{b}^{2}}}},
$$

where $\varphi$ is the angle between $\vec{n}$ and $X$ axis. $\theta_{x}$ and $\theta_{y}$ are the components of $\vec{\theta}$ 
along the $X$ and $Y$ axes. Therefore, Equation (3) can be rewritten as,

$$
u_{\vec{p}_{i} \vec{p}_{j}}(\vec{p})=\min _{t \in[0,1]}\left(t u_{\vec{p}_{i}}+(1-t) u_{\vec{p}_{j}}+\sqrt{\frac{\left(\theta_{x} \cos \varphi\right)^{2}}{r_{a}^{2}}+\frac{\left(\theta_{y} \sin \varphi\right)^{2}}{r_{b}^{2}}}\right) .
$$

As described in Algorithm 1, the AFM algorithm is terminated once the Trial set is empty. After this propagation process, a potential field can be generated with the highest potential value ( $U$ value, the arrival time cost) located at the end point $\vec{p}_{\text {end }}$, while the lowest one is at the start point $\vec{p}_{\text {start }}$ (potential is 0 ). A path can now be extracted from $\vec{p}_{\text {end }}$ to $\vec{p}_{\text {start }}$ following a gradient descent manner in this field.
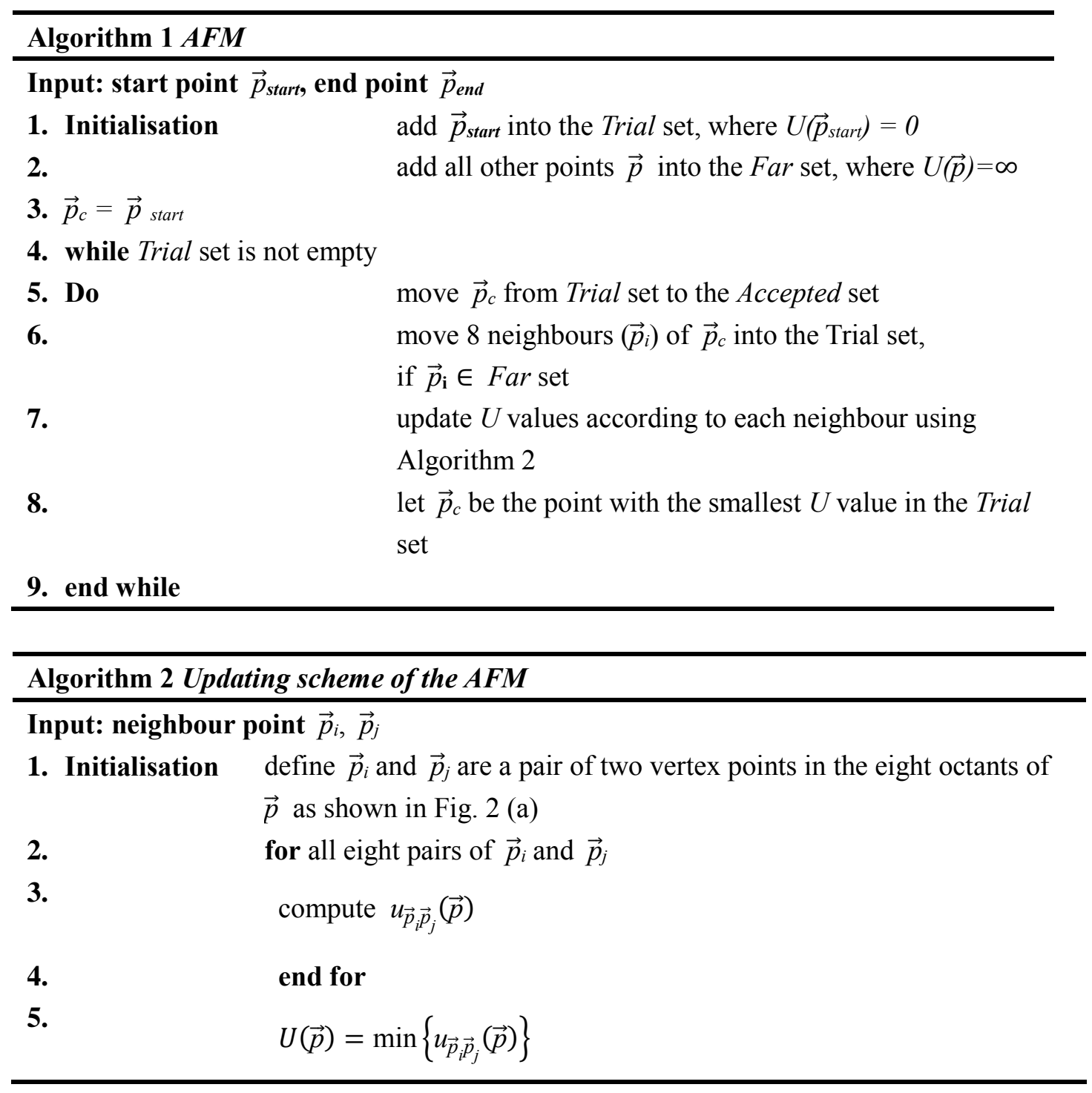


\section{Multi-layered fast marching path planning approach}

\subsection{Algorithm structure}

Fig. 3 outlines the schematic of the multi-layered fast marching (MFM) approach. The main concept is to apply the AFM algorithm on a pre-processed synthetic tensor field map to search for an optimal collision free path. As depicted in Fig. 3, the pre-processed synthetic tensor field map is generated from two-layered information. The first layer is called the Base Layer, which represents the general navigation information and mainly reflects the influence of static obstacles. The base layer has two sub-layers: 1) Attraction layer, on which the goal point of a path is given from the specific mission requirement and 2) Repulsion layer, where static obstacles information, such as islands and coastlines, is provided from the chart.

The second layer is named the Environment Layer. It contains sub-layers of all the information of environmental factors that can affect a USV's voyage, such as currents and wind. All the information (in the base and environment layers) used to search for a path is integrated and converted into a synthetic Tensor Field (TF). Two new operation handlers are developed to help the construction of the synthetic vector field and the tensor field. Details of how to obtain the synthetic TF and the strategies for the optimisation will be illustrated in the following sections. The final optimal path is then generated by applying the AFM algorithm and the gradient descent method onto the synthetic TF.

\subsection{Base vector field}


In general, environmental disturbances, such as currents, are expressed in vector form, so it is necessary to vectorise the base layer information before merging it with any environmental information. A novel FM method based $C$-space vectorisation approach has been developed, named the attractive/repulsive vector field construction process.

\section{Attractive/repulsive vector field construction process}

This process consists of two steps: 1) using the FM method to generate attractive and repulsive potential fields, implicitly reflecting the risk (or influential strength) of the obstacles and attraction from the mission goal point and 2) calculating the gradients of the two potential fields to get the corresponding vector fields. In the first step, the FM method is run twice to obtain the attractive potential field, denoted as $D_{a t t}$, and the repulsive potential field, denoted as $D_{r e p}$, where the calculated distances represent the potential values. $D_{a t t}$ and $D_{\text {rep }}$ can be calculated with expression of:

$$
\begin{gathered}
D_{a t t}(x, y) \leftarrow F M\left(\vec{p}_{\text {goal }}\right), \\
D_{r e p}(x, y) \leftarrow F M\left(\vec{p}_{\text {obs }}, \alpha\right), \quad \alpha \in[0,1]
\end{gathered}
$$

where $\vec{p}_{\text {goal }}$ is the goal point location and $\vec{p}_{\text {obs }}$ is the obstacles' locations in the C-space. $F M(\bullet)$ represents the procedure of running the FM method on the $C$-space, whereas $F M(\bullet, \alpha)$ represents the same FM process but with a new propagation scale limit $\alpha$ introduced. In the latter process, after running the FM method, potential values of all points will be compared with $\alpha$, and any values higher than $\alpha$ will be reset to $\alpha$, which has been mainly introduced to control the influence area of obstacles. For example, as shown in Fig. 4, when $\alpha$ increases, the influence area of obstacle also increases. Also, 
the higher the potential, the safer the position is.

Fig. 5(a) depicts a simulated environment with two small islands located between a channel, where the start and goal points are represented by a shaded red circle and a red star respectively, and the obstacle areas are depicted in black. The generated $D_{\text {att }}$ and $D_{\text {rep }}$ are shown in Figs. 5(b) and (c), where the greater the distance to the goal point (or obstacle), the higher the potential.

In the second step of the attractive/repulsive vector field construction process, the $D_{\text {att }}$ and $D_{\text {rep }}$ are vectorised by calculating the gradients as:

$$
\begin{gathered}
\left(\boldsymbol{F}_{a t t_{x}}, \boldsymbol{F}_{a t t_{y}}\right)=\nabla\left(D_{a t t}\right)=\left(\frac{\partial D_{a t t}}{\partial x}, \frac{\partial D_{a t t}}{\partial y}\right), \\
\left(\boldsymbol{F}_{\text {rep }_{x}}, \boldsymbol{F}_{r e p_{y}}\right)=\nabla\left(D_{\text {rep }}\right)=\left(\frac{\partial D_{r e p}}{\partial x}, \frac{\partial D_{r e p}}{\partial y}\right) .
\end{gathered}
$$

$\nabla\left(D_{\text {att }}\right)$ and $\nabla\left(D_{\text {rep }}\right)$ are further normalised respectively. In this case, the $\boldsymbol{F}_{\text {att }}$ and $\boldsymbol{F}_{\text {rep }}$ become unit vector fields, which means that every element $\left(F_{x}, F_{y}\right)$ in the $\boldsymbol{F}_{\text {att }}$ and $\boldsymbol{F}_{\text {rep }}$ satisfies:

$$
F_{x}^{2}+F_{y}^{2}=1
$$

\section{Base vector field construction process}

In Song et al. [2015], the base vector field $\left(\boldsymbol{F}_{\text {base }}\right)$ is obtained by adding the $\boldsymbol{F}_{\text {att }}$ and $\boldsymbol{F}_{\text {rep }}$ together. However, to simplify the procedures it does not consider the influential strength at each point on the $C$-space. Therefore, in this paper the $\boldsymbol{F}_{\text {base }}$ is generated as a weighted combination of $\boldsymbol{F}_{\text {att }}$ and $\boldsymbol{F}_{\text {rep }}$ as shown in Fig. 5(f), given by:

$$
\boldsymbol{F}_{\text {base }}=\boldsymbol{F}_{\text {att }} \times D_{\text {att }}+\boldsymbol{F}_{\text {rep }} \times\left(1-D_{\text {rep }}\right),
$$

where $\boldsymbol{F}_{\text {att }} \times D_{\text {att }}$ and $\boldsymbol{F}_{\text {rep }} \times\left(1-D_{\text {rep }}\right)$ represent the attractive forces pointing towards the 
mission goal point as shown in Fig. 5(d); and the repulsive forces from the obstacles as shown in Fig. 5(e), where $\alpha=0.3$.

\subsection{Environment vector field}

The predicted global ocean environmental data can be obtained from online resources such as the National Centre for Atmospheric Research (NCAR) and THREDDS data server (TDS). The surface currents vectors are provided as the North/East orthogonal vector components with the information for a particular time is stored in a NetCDF data format. However, as the data is obtained from the satellites observation, the highest precision is $2 \mathrm{~km}$ (Saha et al. [2011]). Because the domain of interest in this paper is within the $5 \mathrm{NM}$ region (based on the effective range of most modern navigation radars and identification devices), the precision of the online data becomes relatively low and not suitable for this research. Simulated current information is therefore adopted in this paper.

To simulate the surface currents with complex spatial variability, the FM method is applied to generate an isotropic power spectrum. Fig. 6(a) shows a 'gyre' flow generated with randomly selected centres' positions $\left(\vec{p}_{c o}\right)$. The central difference method and normalisation are then applied to calculate the two orthogonal components, denoted as $V_{u}$ and $V_{v}$, of the 'gyre' flow and form the flow vector field, given by:

$$
\begin{gathered}
\boldsymbol{F}_{\text {env }}=\boldsymbol{V}_{u}+\boldsymbol{V}_{v}, \\
\boldsymbol{V}_{u}(x, y)=\nabla\left(D_{u}\right)=\left(\frac{\partial D_{u}}{\partial x}, \frac{\partial D_{u}}{\partial y}\right),
\end{gathered}
$$


where $\frac{\partial D_{u}}{\partial x}$ has the similar expression with $\frac{\partial D_{u}}{\partial y}$, and is written as:

$$
\frac{\partial D_{u}}{\partial x}=A^{*}\left\{\begin{array}{lr}
\left(\frac{D_{u}(x+1, y)-D_{u}(x-1, y)}{2 h}\right), & x=2,3, \ldots, N ; y=1,2, \ldots, M \\
\frac{\left(D_{u}(x+1, y)-D_{u}(x, y)\right)}{h}, & x=1 ; y=1,2, \ldots, M, \\
\frac{\left.\left(D_{u}(x, y)-D_{u}(x-1, y)\right)\right)}{h}, & x=N ; y=1,2, \ldots, M
\end{array}\right.
$$

where $N$ and $M$ represent the number of the grids along the $X$ and $Y$ axes respectively, and $h$ is the step size. $A$ is a matrix having the same dimensions as $\boldsymbol{V}_{u}$. The values of $A$ depend on surface currents data and determines the strength of the simulated environmental disturbance.

\subsection{Synthetic vector field}

Once the $\boldsymbol{F}_{\text {base }}$ and $\boldsymbol{F}_{\text {env }}$ are obtained, the final synthetic vector field $\left(\boldsymbol{F}_{\text {syn }}\right)$ is calculated as a function of $\boldsymbol{F}_{\text {base }}$ and $\boldsymbol{F}_{\text {env }}$ with expression of:

$$
\boldsymbol{F}_{\text {syn }}=\beta_{1} \boldsymbol{F}_{\text {env }}+\beta_{2} \boldsymbol{F}_{\text {base }},
$$

where $\beta_{1}$ and $\beta_{2}$ are two field weightings for $\boldsymbol{F}_{\text {env }}$ and $\boldsymbol{F}_{\text {base }}$ respectively with the constraint of $\beta_{1}+\beta_{2}=1$. According to Equation (17), the $\boldsymbol{F}_{\text {syn }}$ generated from Fig. 5(f) and Fig. 6(a) is shown in Fig. 6(b). The values of $\beta_{1}$ and $\beta_{2}$ are determined based upon a 4-regime risk strategy, which will be explained in the next subsection.

\subsection{Operation handlers}

As illustrated in Section 2, the energy cost and the obstacles influence are two of the main concerns when considering the environmental influence in path planning. Therefore, to address these two problems, two operation handlers are specifically 
introduced into the MFM approach: one is called the anisotropic ratio $(A R)$, and the other is the field weightings $(\beta)$.

\section{Anisotropic ratio}

In the MFM approach, using the concept proposed in Section 3.2, $A R$ is introduced to control the angle between the vehicle's heading and the local synthetic vector to make the generated trajectory follow the vector's guidance. $A R$ can be expressed as:

$$
A R=\frac{\left\|\vec{r}_{b}\right\|}{\left\|\vec{r}_{a}\right\|}, \quad A R \in[0,1]
$$

where $\vec{r}_{a}$ represents the major axis of the local ellipse frame, with $\left\|\vec{r}_{a}\right\|$ equalling to the major radius of the local tensor.

The value of $A R$ ranges from 0 to 1. Explicitly, the tensor shape changes with the value of $A R$, as shown in Fig. 7. Implicitly, the $A R$ has an effect on the orientation guidance of the vector field of interest as shown in Fig. 8. It can be seen that when $A R=1$, the MFM method ignores the vector field guidance. When $A R$ tends to 0 , the influence of the orientation guidance increases. However, it can be observed that when the $A R$ value is below 0.04 , the initial part of the trajectory does not follow this trend, as shown in Fig. 8(h) and (i). This is because when the value of $A R$ is relatively small, the resolution of points in the Far set (refer to Algorithm 1) is low compared to those points in the Accepted set.

\section{Field weightings}


The field weightings are the parameters representing the weightings of the environmental influence $\left(\beta_{1}\right)$ and the obstacle influence $\left(\beta_{2}\right)$. To apply the MFM path planning approach into dynamic path planning, where the surface currents change with time, the specific values of $\beta_{1}$ and $\beta_{2}$ need to be adaptively obtained based upon the real environment. To specify the values of $\beta_{1}$ and $\beta_{2}$, a new risk analysis scheme, named the 4-regime risk strategy, has been proposed. The schematic of the 4-regime risk strategy is depicted in Fig. 9. A risk domain is constructed to represent the relationship between the obstacles influences and the environmental influences.

Two risk coefficients termed $\min \left(d\left[\vec{p}, \vec{p}_{o b s}\right]\right)$ and $E(\vec{p})=\left|V_{u} \cdot V_{v}\right|$ are introduced and used in Equation (19) to (22) to evaluate mission priority based upon risk assessment. The $\min \left(d\left[\vec{p}, \vec{p}_{\text {obs }}\right]\right)$ indicates the minimum distance between the USV position $(\vec{p})$ and all obstacles $\left(\vec{p}_{o b s}\right)$, and the $E(\vec{p})=\left|V_{u} \cdot V_{v}\right|$ is defined as the local surface currents strength at $\vec{p}$. According to the risk coefficients, the risk domain is divided into four regimes and the values of $\beta_{1}$ and $\beta_{2}$ are defined as follows.

1) Regime I $\left(R_{\beta_{1}, \beta_{2}}^{1}\right)$ :

$$
R_{\beta_{1}, \beta_{2}}^{1} \in\left\{\vec{p} \mid d_{S A}<\min \left(d\left[\vec{p}, \vec{p}_{o b s}\right]\right)<\frac{1}{2} r n g(o b s) \& \min (E)<E(\vec{p})<\frac{1}{2} r n g(E)\right\}
$$

2) Regime II $\left(R_{\beta_{1}, \beta_{2}}^{2}\right)$ :

$$
R_{\beta_{1}, \beta_{2}}^{2} \in\left\{\vec{p} \mid d_{S A}<\min \left(d\left[\vec{p}, \vec{p}_{o b s}\right]\right)<\frac{1}{2} r n g(o b s) \& \frac{1}{2} r n g(E)<E(\vec{p})<\max (E)\right\},
$$

3) Regime III $\left(R_{\beta_{1}, \beta_{2}}^{3}\right)$ :

$$
R_{\beta_{1}, \beta_{2}}^{3} \in\left\{\vec{p} \mid \frac{1}{2} r n g(o b s)<\min \left(d\left[\vec{p}, \vec{p}_{o b s}\right]\right)<\max \left(d\left[\vec{p}, \vec{p}_{o b s}\right]\right) \& \min (E)<E(\vec{p})<\frac{1}{2} r n g(E)\right\},
$$


4) Regime IV $\left(R_{\beta_{1}, \beta_{2}}^{4}\right)$ :

$$
R_{\beta_{1}, \beta_{2}}^{4} \in\left\{\vec{p} \mid \frac{1}{2} r n g(o b s)<\min \left(d\left[\vec{p}, \vec{p}_{o b s}\right]\right)<\max \left(d\left[\vec{p}, \vec{p}_{o b s}\right]\right) \& \frac{1}{2} r n g(E)<E(\vec{p})<\max (E)\right\},(
$$

where $r n g(o b s)$ is the obstacle range between $d_{S A}$ and $\max \left(d\left[\vec{p}, \vec{p}_{o b s}\right]\right) . d_{S A}$ is the minimum distance that the USV should keep away from the obstacle (shown in Fig. 10). $\max \left(d\left[\vec{p}, \vec{p}_{o b s}\right]\right)$ is the maximum distance from $\vec{p}$ to $\vec{p}_{\text {obs. }} \operatorname{rng}(E)$ is the surface currents flow range between the $\min (E)$ (i.e. the minimum value of $E(\vec{p}))$ and the $\max (E)$ (i.e. the maximum value of $E(\vec{p}))$ in the framework.

In Fig.9 each regime is used to assess the combined environmental and obstacle influence. For example, when the two risk coefficients are located in Regime I, L/H, it represents low environmental influence and high obstacle influence. It can be observed that in Regime I and II, the mission priority is given to obstacle avoidance. In Regime $\mathrm{IV}$, the priority is to follow the surface currents direction to save energy. While in Regime III and on the two boundaries (green lines), the priorities of obstacle avoidance and energy saving are the same, and the USV is in a safe voyage mode.

Based on these four regimes, $\beta_{1}$ and $\beta_{2}$ are calculated as shown in Table I, with the constraint $\beta_{1}+\beta_{2}=1$. Particularly, when the two risk coefficients lie on the green lines in Fig. $9\left(\min \left(d\left[\vec{p}, \vec{p}_{o b s}\right]\right)=\frac{1}{2} r n g(o b s)\right.$ and $\left.E(\vec{p})=\frac{1}{2} r n g(E)\right), \beta_{1}=\beta_{2}=0.5$. 


\section{Simulations and results}

The proposed MFM approach has been tested and verified via three different simulations in a static environment subject to variant and invariant surface currents as:

1) Path planning in a time-invariant environment with the purpose to evaluate the performance of the orientation guidance capability of the MFM approach (Section 5.1).

2) Path planning controlled by operation handlers to validate the impacts of the two operation handlers, namely anisotropic ratio $(A R)$ and field weighting $(\beta)$ (Section 5.2).

3) Path planning in a time-variant environment to validate the dynamic path planning capability of the MFM method. Two different scenarios have been considered in this simulation: a) surface currents are stationary but the USV keeps changing positions (Section 5.3.1); b) surface currents vary with time and the USV keeps moving along the planned trajectory (Section 5.3.2).

The path planning methods have been coded in MATLAB and simulations are run on the computer with a Pentium i7 3.4 GHz processor and 8GB of RAM. The simulation area is presented in Fig. 11(b) with dimension of $2 * 2 \mathrm{~km}$, which is rasterised to a uniform, $500 * 500$ grid map (the size of each grid is $1 * 1$ pixel, and 1 pixel equals to 4 $\mathrm{m})$. The magnitude range of the currents velocity is between 0 to $1 \mathrm{~m} / \mathrm{s}$. To quantitatively assess the performances of the generated paths, three types of evaluations are considered:

- the minimum distance to the obstacles, denoted as min_Obs, is calculated as: 


$$
\min \_ \text {Obs }=\min \left[d\left(\overrightarrow{p, \vec{p}_{\mathrm{obs}}}\right)\right]
$$

where $\vec{p}$ is the USV current position, $\vec{p}_{\text {obs }}$ are the positions of all obstacles in the environment and $d\left(\overrightarrow{p,} \vec{p}_{\text {obs }}\right)$ returns the distances between $\vec{p}$ and $\vec{p}_{\text {obs }} \cdot \min [\bullet]$ is the process that extracts the minimum value from $d\left(\overrightarrow{p,} \vec{p}_{o b s}\right)$.

- the total distance cost can be represented as the cumulated distance over the entire running time, and can be expressed as:

$$
D_{\text {cum }}=\sum_{i=1}^{n} d_{i}
$$

where $d_{i}$ is the length of $i$ th path segment during time $\Delta \mathrm{t}, n$ is the total number of path segments.

- the total energy cost, which is the sum of the power consumption at each position, can be expressed in the form of:

$$
W=\sum_{i=1}^{n} W_{i}
$$

where $n$ is the number of points along the trajectory. Since USVs are built in different types with varying power sources, ideally the energy cost should be calculated according to each individual propulsion system, which is difficult to measure in this paper. An alternative is to express the total energy cost by calculating the added energy required to overcome the drag force generated by the surface currents along the trajectory, and such a force can be expressed as the angle between the USV velocity and current velocity (Alvarez et al. [2004]). Therefore, the power consumption $\left(W_{i}\right)$ at each position is calculated using the Equation (26): 


$$
W_{i}=\frac{\rho J}{c}
$$

where $\rho$ is the water density, $c$ is the nominal speed of the USV and $J$ is the integration of the relative velocity of USV to the surface currents, expressed as:

$$
J=\iint\left\|c e_{\vec{p}}-\boldsymbol{F}_{e n v}\right\| d x d y
$$

where $e_{\vec{p}}$ represents the unit vector of USV heading at point $\vec{p}$.

In addition, before undertaking the simulations, the following assumptions have been made:

1) The hydrodynamic forces have not been considered. The USV is configured as a mass point, and the influence of the forces acting on the USV is regarded as the effect of the generated vector at each grid point.

2) In order to facilitate the implementation of the gradient descent method to effectively search for the path, the mission goal point is set as the start point of the anisotropic fast marching (AFM) method so that the mission goal point has the lowest potential. Then, the gradient descent method is used to search for the path from the mission start point towards the goal point.

3) The USV is operating at a constant speed of $1.5 \mathrm{~m} / \mathrm{s}$ over the ground for dynamic path planning scenarios.

4) A safe margin has been defined as $20 \mathrm{~m}$, which is considered to be sufficient for a small size USV to avoid obstacles. 
5) The water in the environment is assumed to have a constant density, which means that $\rho$ is constant. As the USV speed $c$ in Equation (26) is constant, the total energy cost can be evaluated as the sum of $W_{i}$ that is a function of $J$. The total energy cost, can, therefore be calculated in the expression of:

$$
W=C \sum_{i=1}^{n} J_{i}
$$

where $C=\rho / c$.

\subsection{Path planning in a time-invariant environment}

The conventional FM algorithm is run first. The generated potential field is shown in Fig. 11(a), where the colour varies from blue to red, representing different potential values from low to high ( 0 to 1 as shown in the bar chart of Fig. 11(a)). It also can be observed that the goal point has the lowest potential. As discussed in Section 3, the 'wave' propagates in a circular shape and does not enter the obstacles' area, which has been pre-distinguished using the Otsu method (Otsu [1975]). The generated off-line trajectory is plotted as the blue line in Fig. 11(b). The trajectory is able to successfully avoid obstacles. However, as the primary aim of the FM method is to achieve the minimum distance cost, the generated path stays too close to the obstacle, which is not the best option for the USV.

The MFM algorithm is then run in the same environment using the same mission start and goal points. The generated potential field and trajectory are shown in Fig. 12(a) and Fig. 12(b). From the potential field, it can be observed that the propagation 'wave' does 
not follow a circular shape but instead trends towards a 'synthetic' direction based on the synthetic tensor field as shown in the shaded red ellipses in Fig. 12(a). The green line shown in Fig.12(b) is the off-line path planning result using the MFM method. It can be observed that as the green path needs to follow the vector's direction, the initial section of the path has been calculated towards northeast, which is different from the blue path. However, similar to the FM method's result, the last section of the green trajectory is also too close to the island obstacle with potential collision risk. This is because the values of the two operation handlers are set as 0.5 , which means that the obstacle influence has the same weighting as the surface currents influence.

To further compare the two results, quantitative evaluations of the two paths are represented in Fig. 13. Fig. 13(a) compares the minimum obstacle distances - the distance of each coordinate (CO) to the closest obstacle - along the blue and green trajectories. It can be seen that the minimum obstacle distances for both paths are above 0, which proves that both the trajectories have no collisions with the obstacles. However, from CO number 390 to 690 and 800 to 950 for the blue path; and from CO 1450 to 1680 for the green path, the minimum obstacle distances are both below $20 \mathrm{~m}$, which is the predefined minimum safe margin. These indicate that there is potential for collisions when the USV is tracking along these sections of the two trajectories. In addition, the total number of $\mathrm{CO}$ for the green path is 2030 , which is $33.55 \%$ larger than the blue path (the number of $\mathrm{CO}$ is 1520). When comparing the total distance cost (shown in Fig. $13 \mathrm{~b}$ ), the green path is $33.98 \%$ greater than the blue path. The two increasing rates are 
almost the same, but the green path consumes less energy cost (46.27\% less) than the blue path as shown in the bar chart in Fig. 13(c). These data indicate that by tracking the generated trajectory from the MFM method can save on the energy cost while keeping the distance cost as low as possible. In terms of the computational time, it takes $0.13 \mathrm{~s}$ for the FM method to generate the blue path and $0.85 \mathrm{~s}$ for the MFM method to calculate the green path. Although the MFM method takes a longer time than the FM method, the MFM method is still very fast.

\subsection{Path planning controlled by operation handlers}

\subsubsection{Anisotropic ratio variation}

To verify the effects of the anisotropic ratio $(A R)$ on the generated trajectory in the presence of obstacles, different $A R$ values are tested in the same environment with the same mission start and goal points in Section 5.1. The path planning results and the performance evaluations are shown in Fig. 14 and Fig. 15. The green line in Fig. 14 is the calculated path when $A R$ is 0.5 , the light blue line is the path result when $A R$ is increased to 0.8 and the magenta line is the generated path when $A R$ is reduced to 0.2 . It can be seen from Fig. 15(a) that all the three paths have no collisions with obstacles, but there are some parts that fall below the safety margin so there is an increased collision risk. In Fig. 15(b) and (c), it shows that the magenta path has the least distance cost $(958.92 \mathrm{~m})$ and the best performance to follow the currents vectors guidance $(90.27$ degrees). This indicates that when controlling the $A R$ values, the optimisation performance of the generated trajectory has the same trend as explained in Section 4.5, 
namely, as $A R$ reduces, the energy cost reduces. The average computational time of the three tests is $0.83 \mathrm{~s}$.

\subsubsection{Field weighting variation}

The effects of the field weightings $\beta_{1}$ and $\beta_{2}$ ) on the generated trajectories are shown in this section. Two simulations are undertaken when $\beta_{2}$ equals $0.5\left(\beta_{1}=1-0.5=0.5\right)$ and $0.8\left(\beta_{1}=1-0.8=0.2\right)$. The generated paths are depicted in the green line and magenta line in Fig. 16(a) and (b) respectively. It can be seen that after increasing the obstacle influence $\left(\beta_{2}\right)$, the magenta path keeps a further distance away from the obstacles. Comparing the performances of these two paths, shown in Fig. 17(a)-(c), it can be seen that although the magenta path has longer distance cost (45.57\% increased) and more energy cost $(12.22 \%$ increased), the distances to obstacles have been increased significantly as is clearly shown. This indicates that as $\beta_{2}$ increases, the path becomes safer. The average computational time of generating the green and magenta paths is 0.84 s.

\subsection{Path planning in a time-variant environment}

Two simulations have been undertaken to validate the dynamic path planning ability of the MFM method. In these two tests, the values of $\beta_{1}$ and $\beta_{2}$ are calculated according to Table I. Based on the simulation results shown in Section 5.2.1, the value of $A R$ is set to be 0.04 in regimes III and IV of the 4-regime risk strategy, while in regimes I and II the value of $A R$ is set to the same value as $\beta_{2}$. The path recalculation process is executed 
every 1 time step $(\Delta t=1)$ with a step size of $320 \mathrm{~m}$. At each time step, the environment is re-analysed and a new path is generated on the updated synthetic environment framework.

\subsubsection{USV position changes with time}

The aim of the first simulation is to test the scenario where the USV voyage is in an environment with stationary surface currents. The launch and goal points for the USV are marked as a shaded red circle and a star in Fig. 18 with coordinates $(76,278)$ and $(234,349)$, respectively. The dynamic path planning results are shown in Fig. 18. The blue arrows represent the orientations and strengths of the synthetic vector fields at different time steps. The blue line is the generated path when the USV is at the launch point. The green lines represent the updated path results and the magenta lines are the USV tracked trajectory. The black circles represent the start point of each recalculation process. To further clarify and demonstrate the capability of the proposed algorithm, instantaneous values of the two operation handlers $(A R$ and $\beta)$ are represented at the right bottom section of each figure.

Since the USV is close to the obstacle along the initial section of the trajectory (as shown in Fig. 18(a) and (b)), the mission priority has been given to collision avoidance rather than energy saving by using the 4-regime risk strategy analysis, and $\beta_{2}$ is therefore assigned a higher value than $\beta_{1}$. Using these two values and following corresponding trajectories, the magenta trajectory in Fig. 18(c) shows that the USV is able to keep a 
safe distance away from all obstacles en route. However, as the USV moves farther away from the obstacles as shown in Fig. 18(c), (d) and (e), the mission priority is now changed to energy saving and the $A R$ is consequently calculated to be equal to 0.04 with $\beta_{2}$ being reduced accordingly. It can be observed that the re-planned path (green path) follows the orientation guidance of the blue arrows closely. It however should be noted that the green path cannot always guarantee the safety of the USV. For example, from Fig. 18(f), it can be seen that by following the green path, the USV could potentially collide with the obstacle. To ensure the safety of the navigation, $\beta_{2}$ is therefore increased again to push the trajectory away from the obstacles, as shown from Fig. 18(g) to (i). The average computational time of the trajectory updating process, including the environment framework reconstruction and trajectory calculation, is around $0.91 \mathrm{~s}$.

\subsubsection{USV position and currents change with time}

The second simulation shows how the dynamic path planning is undertaken in the same environment area in Section 5.3.1 but against dynamic surface currents. The results are shown in Fig. 19 where the launch point $(90,279)$ and goal point $(323,356)$ are depicted as a shaded blue circle and star respectively. In the free space, the black arrows represent the corresponding synthetic vector field. The red and yellow colours represent the surface currents coming from the east and west respectively with a more intense colour representing stronger surface currents. The blue line shows the generated path when the USV is located at the launch point. The green lines are the renewed path results after applying the MFM method upon the updated synthetic vector field and location of the 
start point. The instantaneous USV position at different time steps is depicted as black circles. The sequence of black circles represents the USV tracking trajectory.

Similar to the previous simulation, the priority of collision avoidance is higher when the USV moves along the initial part of the trajectory as shown from Fig. 19(a) to (c). Note that comparing Fig. 19(a) and (b), differences can be observed between the green path (re-planned path) and the blue path (initial path). The reason behind this is that as the surface currents coming from the east become stronger near the final section of the blue trajectory, $\beta_{1}$ is being increased ( $A R$ decreased) to follow the guidance of the currents as much as possible. However, as the USV approaches the obstacles, $\beta_{2}$ increases and the last part of the green path restores to the original trend even though the surface currents in Fig. 19(c) are stronger than that in Fig. 19(b). From time steps 11 to 39 as shown from Fig. 19(d) to (h), as the USV moves further away from the obstacles, the green path is updated according to the changing surface currents and follows the orientation guidance of the black arrows. It can be seen from Figs. 19 (f) to (h), as the surface currents strength increases, the green path comes too close to the middle obstacle. To reduce the collision risk, $\beta_{2}$ starts to increase from time step 40 as shown in Fig. 19(i) until the collision risk is eliminated as shown in Fig. 19(k). The average computational time of the trajectory updating at each time step is $0.92 \mathrm{~s}$. 


\section{Conclusions}

A novel multi-layered fast marching (MFM) path planning method has been developed to provide a practical trajectory and been tested. The path can be calculated to accommodate time dependent surface currents and has the advantage that it is adaptable. The algorithm constructs a synthetic environment framework, which consists of a base vector field and an environment vector field. The algorithm evaluates risks from the obstacles and environmental factors and 'intelligently' decides the mission priorities using the attractive/repulsive vector field construction process and the 4-regime risk strategy analysis. The AFM method is selected to search for an optimal path over the synthetic framework.

The proposed algorithm has been validated against the conventional FM method. It shows that the FM method cannot take the ocean currents into account causing the generated path to have high collision risks in some areas. In contrast, the proposed MFM method provides a safer path by using two operation handlers. The path is able to keep a safe distance away from obstacles and saves on the energy cost by following counter-flow areas. In addition, the computational time of the MFM method has been tested to be less than 1 second, which satisfies the general sampling time requirement of the autopilot system of a practical USV (Naeem et al. [2006]).

In terms of future work, the main focus will be on the improvement of the practicability of the algorithm. Currently, surface currents are considered as the only environmental 
influence. Other factors such as wind also need to be taken into account, especially for a small sized vessel such as an autonomous sailboat (Stelzer and Pröll [2008]). Using a similar approach, wind can be converted into vector field and integrated onto the Environment Layer together with other environmental influences. In addition, to assist with the practical application, the proposed algorithm will extract the Base Layer information from the electronic navigational chart such that the practicability of the generated path will be increased. Moreover, when avoiding moving vessels, more feasible and practical manoeuvers should be adopted. COLREGs regulations can be integrated into the algorithm so that the most appropriate evasive action can be taken by the USV according to different encounter situations. When considering the regulations, a scheme will be developed to evaluate and prioritise the mission optimisation costs. The highest priority should be given to the COLREGs compliant collision avoidance with the distance cost having lower priority when applying the path searching algorithm (Tam and Bucknall [2010]). The energy optimisation will only be taken into consideration when the collision risk is cleared.

Apart from the algorithm improvement, the implementation of the MFM on a practical USV will also be investigated. The possible platform will be Springer USV, which is a low-cost research vessel designed and developed by Plymouth University with the primary aim of undertaking pollutant tracking and environmental monitoring operations (Naeem et al. [2008]). Using the improved navigation guidance and control (NGC) system proposed in Liu et al [2015], Springer is capable of autonomously 
planning the trajectory and robustly following the route based upon the mission requirements. The next step is to replace the current path planning algorithms of the NGC system (mainly the A* and the conventional FM method) with the MFM method to make the vehicle better able to deal with various tidal currents influence. It is expected that using the predicted tidal currents data, Springer can be used to conduct complicated missions in open sea areas.

\section{Acknowledgement}

This work is supported by the ACCeSS group. The Atlantic Centre for the innovative design and Control of Small Ships (ACCeSS) is an ONR-NNRNE programme with Grant no. N0014-03-0160, the group consists of universities and industry partners conducting research into control of small ships.

\section{References}

Agarwal, A. and Lermusiaux, P. F. J. (2011), 'Statistical field estimation for complex coastal regions and archipelagos', Ocean Modelling, 40 (2), 164-89.

Alvarez, A., Caiti, A., and Onken, R. (2004), 'Evolutionary path planning for autonomous underwater vehicles in a variable ocean', IEEE Journal of Oceanic Engineering, 29 (2), $418-429$.

Alves, J., Oliveira, P., Oliveira, R., Pascoal, A., Rufino, M., Sebastiao, L., and Silvestre, C. (2006), 'Vehicle and Mission Control of the DELFIM Autonomous Surface Craft', Control and Automation. MED '06. 14th Mediterranean Conference (IEEE), 1-6. 
Andrews, J. R. (1983), 'Impedance Control as a Framework for Implementing Obstacle Avoidance in a Manipulator', Master's thesis, Massachusetts Institute of Technology, Department of Mechanical Engineering.

Caccia, M., Bono, R., Bruzzone, G., Spirandelli, E., Veruggio, G., Stortini, A. M., and Capodaglio, G. (2005), 'Sampling sea surfaces with SESAMO: an autonomous craft for the study of sea-air interactions', IEEE Robotics \& Automation Magazine, 12 (3), 95-105.

Caselles, V., Kimmel, R., \& Sapiro, G. (1995). ‘Geodesic active contours', Proceedings. IEEE Fifth International Conference on Computer Vision, 694-699.

Chen, D. Z., Szczerba, R. J., and Uhran, J. J. (1995), 'Planning conditional shortest paths through an unknown environment: a framed- quadtree approach', Intelligent Robots and Systems 95. 'Human Robot Interaction and Cooperative Robots', Proceedings. 1995 IEEE/RSJ International Conference on, (3), 33-38.

Ferreira, H., Martins, A., Dias, A., Almeida, C., Almeida, J., and Silva, E. (2006), 'ROAZ Autonomous Surface Vehicle Design and Implementation', Robotica.

Garrido, S., Moreno, L., and Blanco, D. (2008), 'Exploration of a cluttered environment using Voronoi Transform and Fast Marching', Robotics and Autonomous Systems, 56 (12), 1069-1081.

Goldberg, D. E. (1989), 'Genetic algorithms in search, optimization and machine learning', Addison-Wesley, Boston; London, 3(2), 95-99.

Konukoglu, E., Sermesant, M., Clatz, O., Peyrat, J. M., Delingette, H., \& Ayache, N. (2007, 
July). 'A recursive anisotropic fast marching approach to reaction diffusion equation: Application to tumor growth modeling'. In Biennial International Conference on Information Processing in Medical Imaging (pp. 687-699). Springer Berlin Heidelberg.

Kamon, I. and Rivlin, E. (1995), 'Sensory based motion planning with global proofs', IEEE Transactions on Robotics and Automation, (2), 435-40.

Khatib, O. (1986), 'Real-time obstacle avoidance for manipulators and mobile robots', The international journal of robotics research, 5(1), 90-98.

LaValle, S. M. (1998), 'Rapidly-exploring random trees: A new tool for path planning'.

LaValle, S. M. (2006), 'Planning algorithms', Cambridge University Press, New York.

Lin, Q. (2003), 'Enhancement, Extraction, and Visualization of 3D Volume Data'. Linköping Studies in Science and Technology.

Liu, Y., Song, R., \& Bucknall, R. (2015, May). 'A practical path planning and navigation algorithm for an unmanned surface vehicle using the fast marching algorithm'. In OCEANS 2015-Genova. pp. 1-7. IEEE.

Lolla, T., Haley Jr, P. J., and Lermusiaux, P. F. J. (2015), 'Path planning in multi-scale ocean flows: Coordination and dynamic obstacles', Ocean Modelling, 94, 46-66.

Lolla, T., Lermusiaux, P., Ueckermann, M., and Haley, P. (2014), 'Time-optimal path planning in dynamic flows using level set equations: theory and schemes', Theoretical, Computational and Observational Oceanography, 64 (10), 1373-1397.

Mirebeau, J. M. (2014). 'Anisotropic fast-marching on cartesian grids using lattice basis reduction'. SIAM Journal on Numerical Analysis, 52(4), 1573-1599. 
Naeem, W., Sutton, R., \& Chudley, J. (2006, August). 'Modelling and control of an unmanned surface vehicle for environmental monitoring'. In UKACC International Control Conference.

Naeem, W., Xu, T., Sutton, R., \& Tiano, A. (2008). 'The design of a navigation, guidance, and control system for an unmanned surface vehicle for environmental monitoring'. Proceedings of the Institution of Mechanical Engineers, Part M: Journal of Engineering for the Maritime Environment, 222(2), 67-79.

Otsu, N. (1975). 'A threshold selection method from gray-level histograms'. Automatica, 11(285-296), 23-27.

Petres, C., Pailhas, Y., Petillot, Y., and Lane, D. (2005), 'Underwater path planing using fast marching algorithms', Oceans - Europe, 814-19.

Petres, C., Pailhas, Y., Patron, P., Petillot, Y., Evans, J., and Lane, D. (2007), 'Path planning for autonomous underwater vehicles', IEEE Transactions on Robotics, 23 (2), 331-341.

Saha, S., et al. (2011), updated daily. 'NCEP Climate Forecast System Version 2 (CFSv2) 6hourly Products'. Research Data Archive at the National Center for Atmospheric Research, Computational and Information Systems Laboratory. http://dx.doi.org/10.5065/D61C1TXF. Accessed 9 Jul 2015.

Sethian, J. A. (1996). 'A fast marching level set method for monotonically advancing fronts'. Proceedings of the National Academy of Sciences, 93(4), 1591-1595.

Smierzchalski, R. (1999), 'Evolutionary trajectory planning of ships in navigation traffic areas', Journal of marine science and technology, 4 (1), 1-6. 
Stelzer, R., \& Pröll, T. (2008). 'Autonomous sailboat navigation for short course racing'. Robotics and autonomous systems, 56(7), 604-614.

Song, R. (2014). 'Path planning and bi-directional communication for unmanned surface vehicle'. MIDAS technical report. MIDAS MSE.2014.TR.011, MIDAS.

Song, R., Liu, Y., Liu, W., and Bucknall, R. (2015), 'A two-layered fast marching path planning algorithm for an unmanned surface vehicle operating in a dynamic environment', OCEANS 2015 - Genova (Genoa: IEEE), 1-8.

Statheros, T., Howells, G., and Maier, K. D. (2008), 'Autonomous Ship Collision Avoidance Navigation Concepts, Technologies and Techniques', Journal of navigation, 61 (1), $129-142$

Tam, C., \& Bucknall, R. (2010). 'Collision risk assessment for ships'. Journal of Marine Science and Technology, 15(3), 257-270.

Tsitsiklis, J. N. (1995), 'Efficient algorithms for globally optimal trajectories', IEEE Transactions on Automatic Control, 40 (9), 1528-1538.

Wu, P., Xie, S., Luo, J., Qu, D., and Li, Q., 'The USV Path Planning Based on the Combinatorial Algorithm', Rev. Tec. Ing. Univ. Zulia, 38(2015) 62-70

Xu, T., Chudley, J., \& Sutton, R. (2006). 'A fault tolerant multi-sensor navigation system for an unmanned surface vehicle'. IFAC Proceedings Volumes, 39(13), 1503-1508. 
Figures and tables

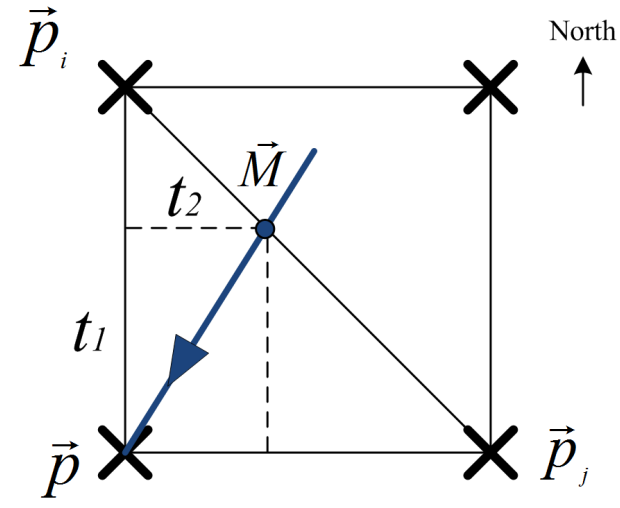

(a)

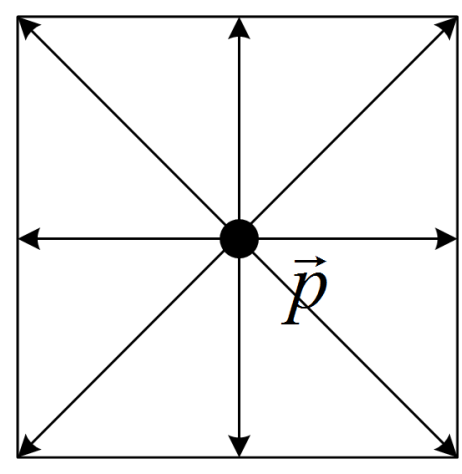

(b)

Fig. 1 (a) the FM method updating scheme. (b) 4-geometry cell connection scheme. 


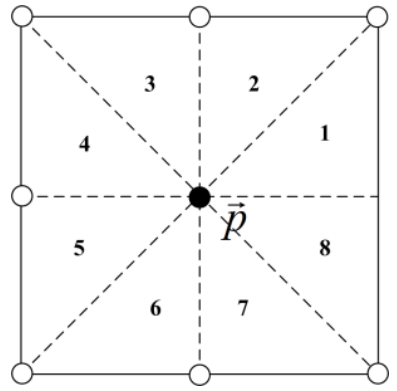

(a)

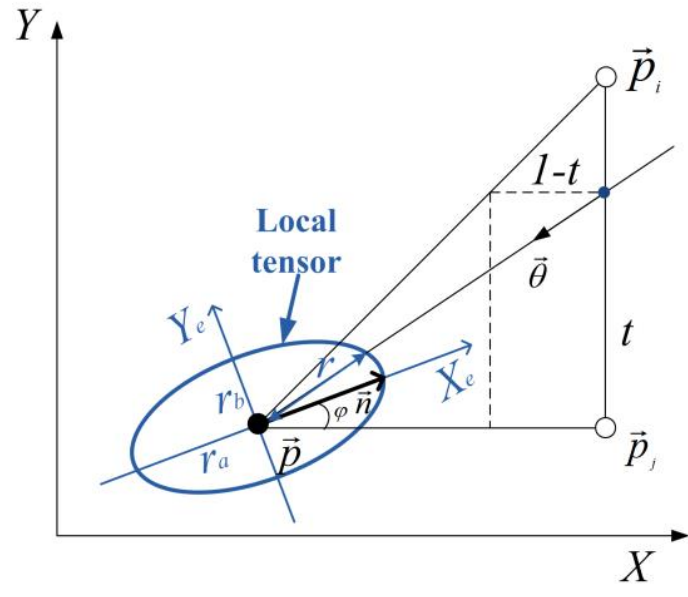

(b)

Fig. 2 (a) Eight octants definition of the anisotropic FM updating scheme. (b) Schematic of calculating arrival time at neighbour point $\vec{p}$. 


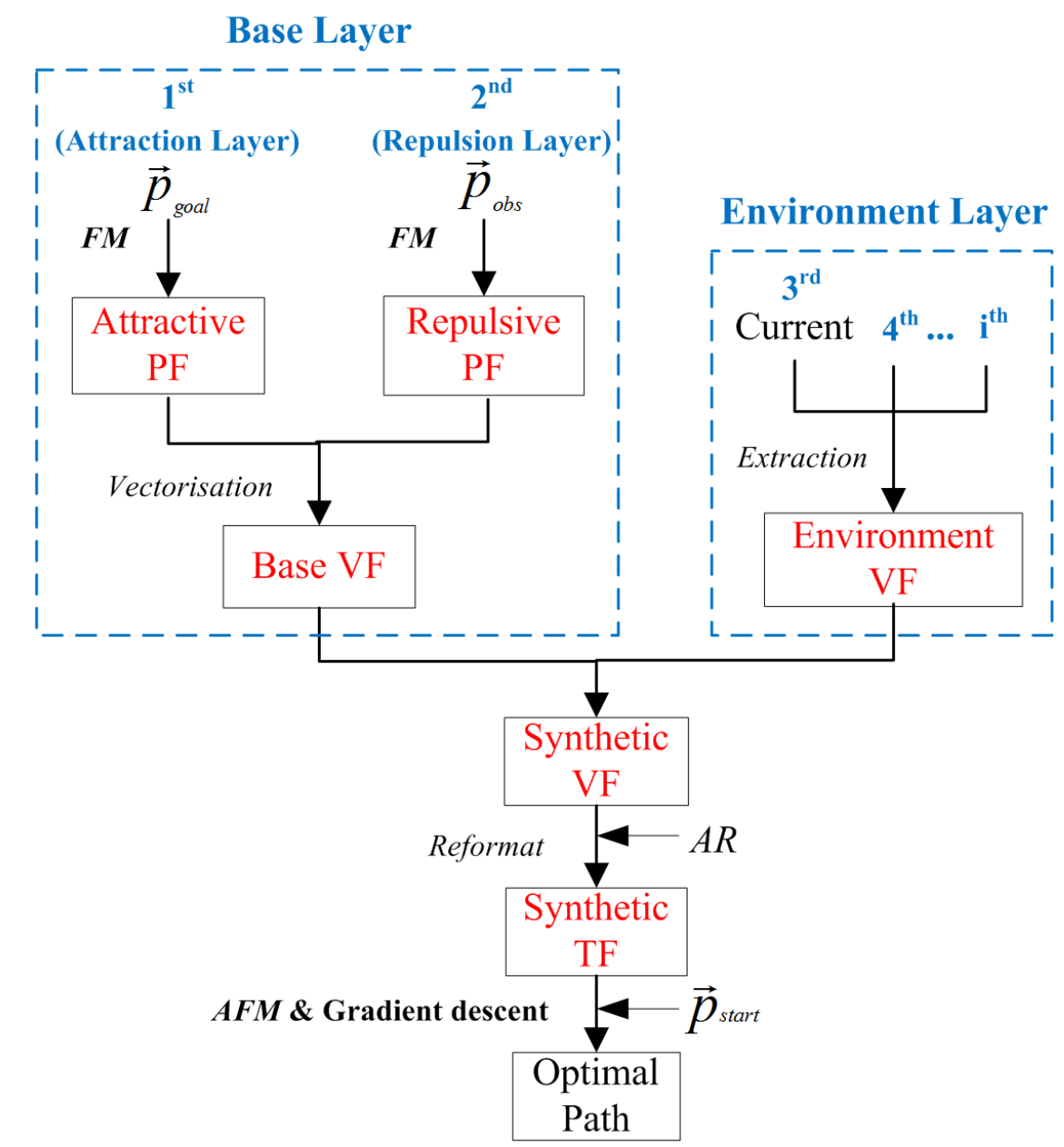

Fig. 3 Schematic of the MFM method. PF and VF are short for potential field and vector field respectively. 


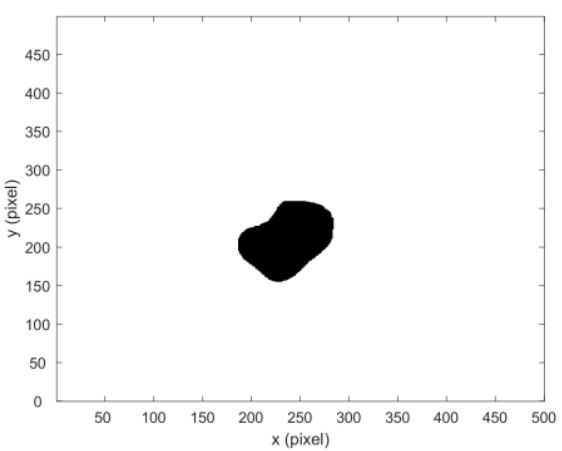

(a)

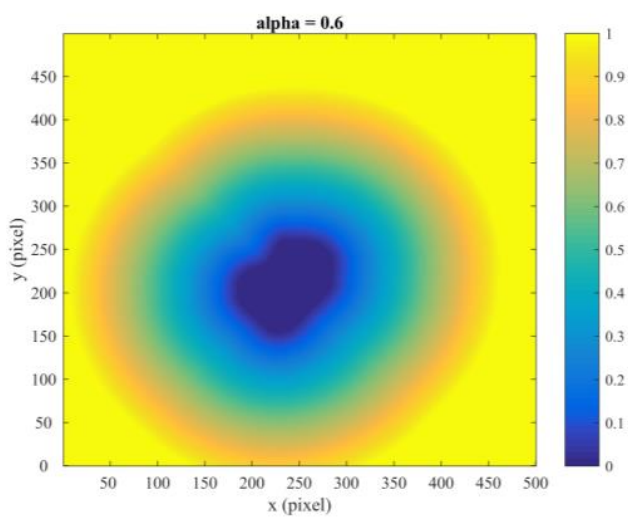

(c)

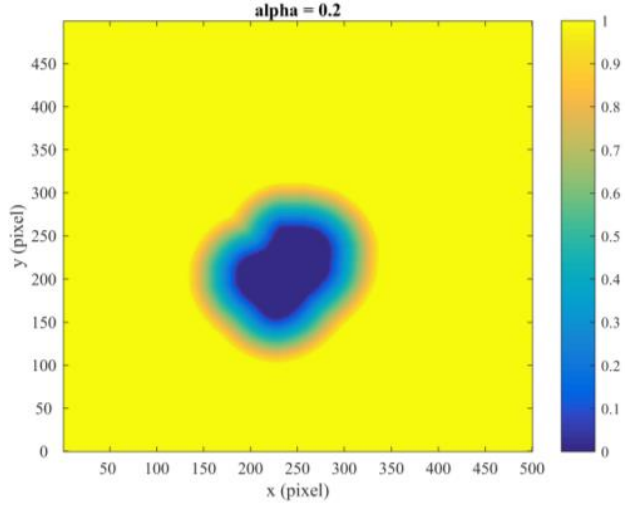

(b)

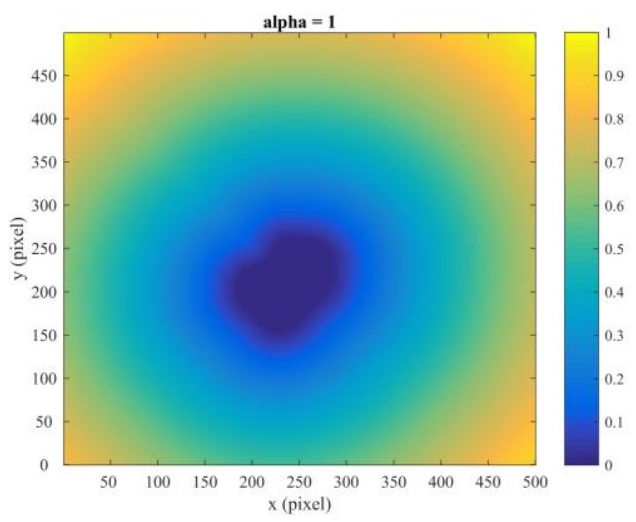

(d)

Fig. 4 Changes of potential field with different $\alpha$ values on a map of 500*500 grids. (a) Grid map with one obstacle. (b), (c) and (d) are the potential fields when $\alpha=0.2,0.6$ and 1, respectively. The colour from blue to yellow represents the influence strength of the obstacle, where blue indicates the highest influence and yellow means there is no influence. 


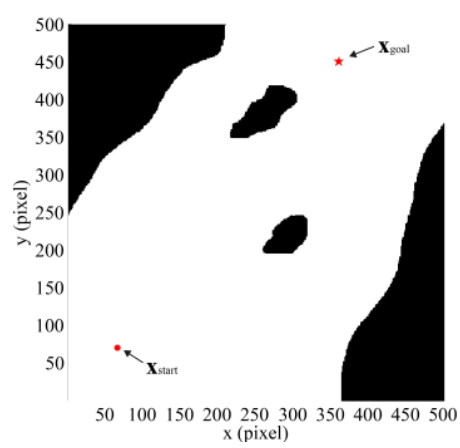

(a)

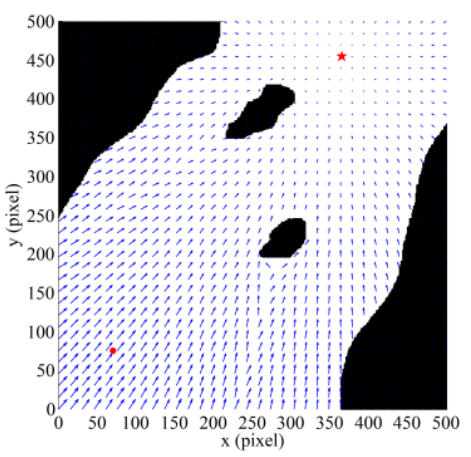

(d)

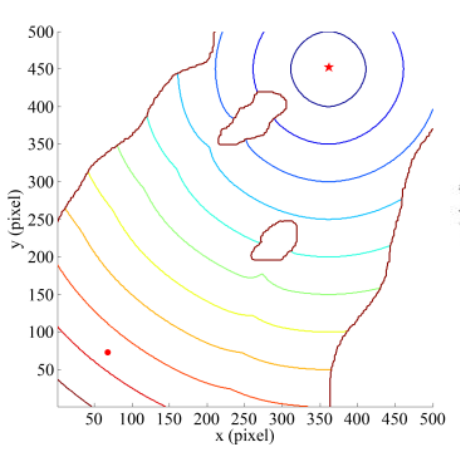

(b)

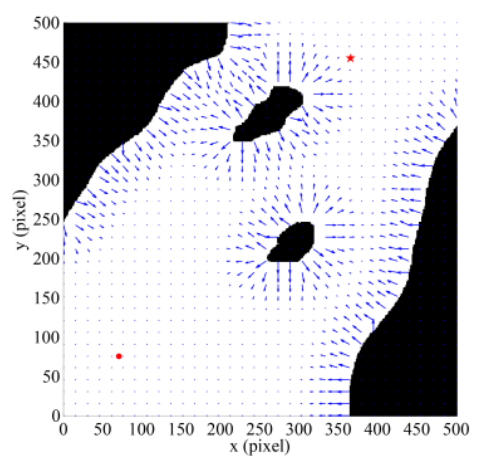

(e)

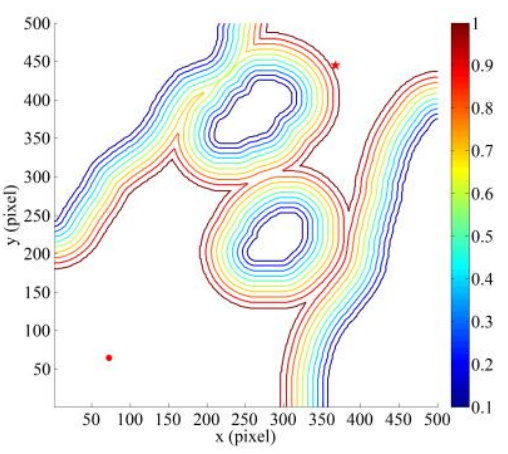

(c)

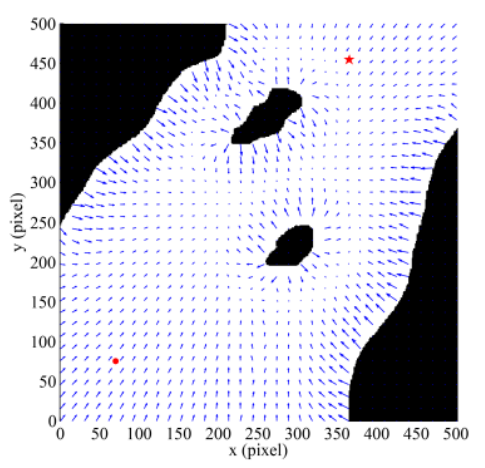

(f)

Fig. 5(a) Map of the self-constructed environment, with dimension of 500*500 grids. Each grid size is 4 $\mathrm{m}$. The red dot is the $\vec{p}_{\text {start }}$ with location at $(66,71)$ in the environment, and the red star is the $\vec{p}_{\text {goal }}$ located at $(361,450)$. (b) The generated attractive potential field, $D_{\text {att }}$, by applying the FM method from the $\vec{p}_{\text {goal }}$. (c) The generated repulsive potential field, $D_{\text {rep }}$, by applying the FM method from the $\vec{p}_{\text {Obs }}$. The coloured bar at right depicts the range of potential values. (d) The corresponding attractive forces $\left(\boldsymbol{F}_{\text {att }} \times D_{\text {att }}\right)$ of $D_{\text {att }}$. The length of the arrow represents the magnitude of force. (e) The repulsive forces $\left(\boldsymbol{F}_{\text {rep }} \times\left(1-D_{\text {rep }}\right)\right)$ of $D_{\text {rep }}$ with $\alpha=0.3$. (f) The generated base vector field $\left(\boldsymbol{F}_{\text {base }}\right)$. 


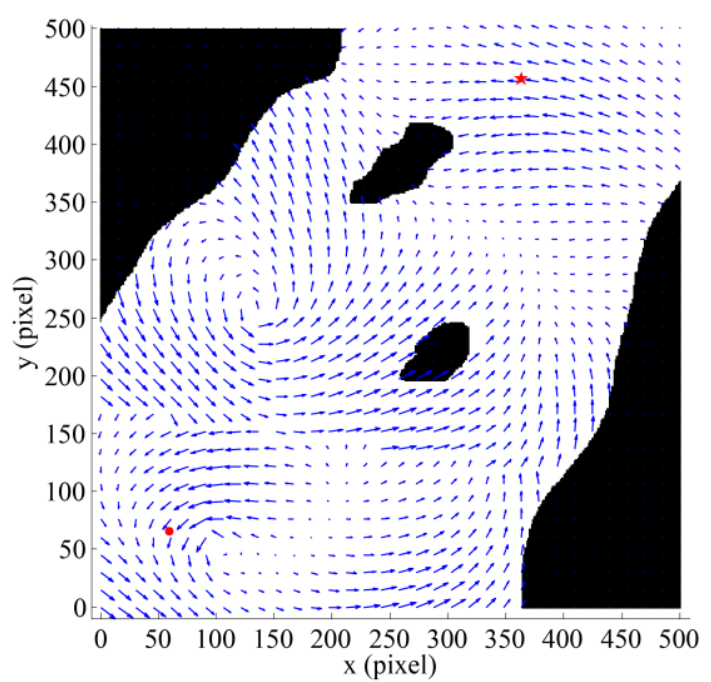

(a)

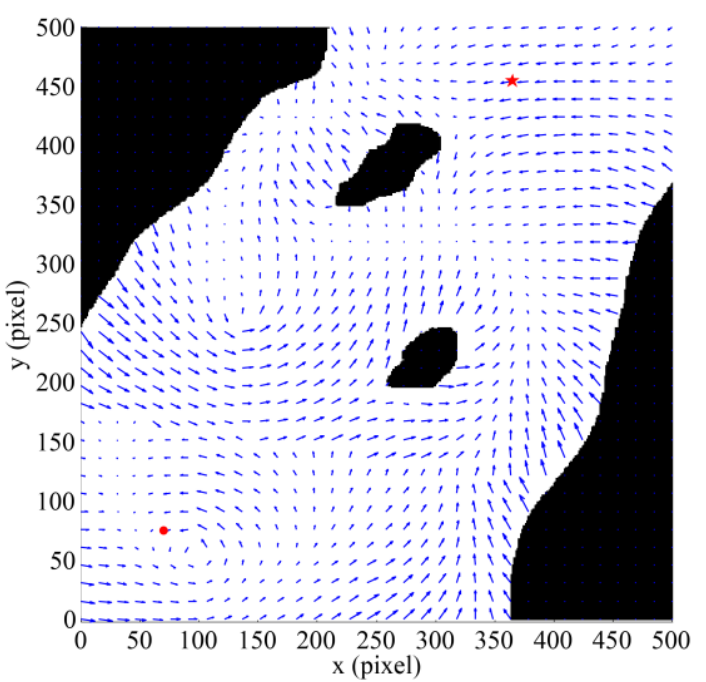

(b)

Fig. 6 (a) Simulated surface currents environment $\boldsymbol{F}_{\text {env }}$ (b) Generated $\boldsymbol{F}_{\text {syn }}$ from Fig. 5(f) and Fig. 6(a) with equal field weightings, namely $\beta_{1}=\beta_{2}=0.5$. 


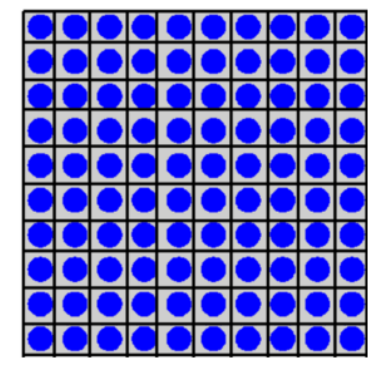

(a)

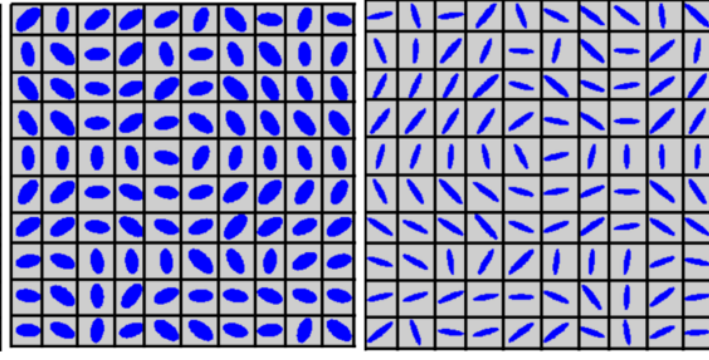

(b)

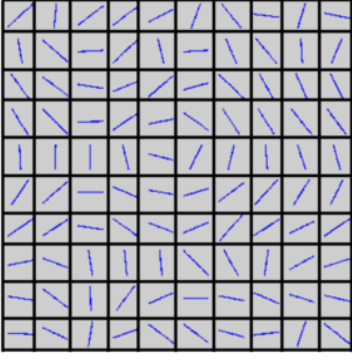

(d)

Fig. 7 Changes of a tensor field with different values of $A R$ on a map of $10 * 10$ grids. The shape of each tensor is represented in blue. (a) When $A R=1$, the tensor is a shaded circle. (b) When $A R=0.5$, the tensor is a shaded ellipse. (c) When $A R=0.2$, the tensor is a slim shaded ellipse. (d) When $A R=0.01$, the tensor is close to a shaded line. 


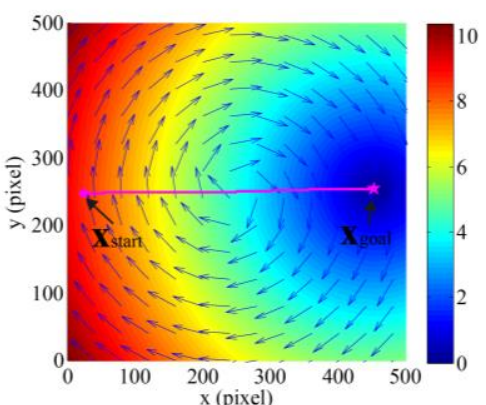

(a)

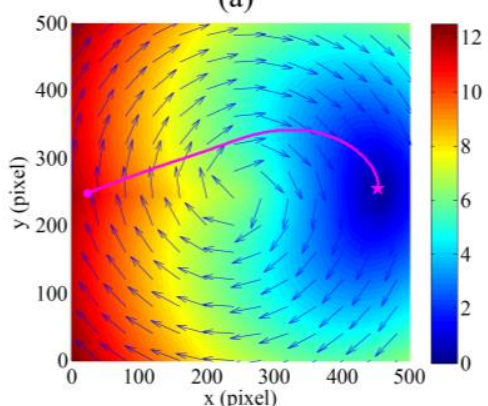

(d)

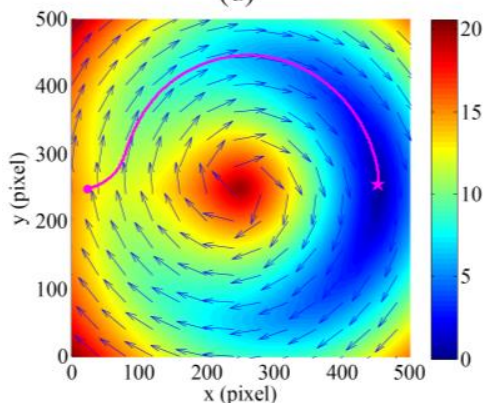

(g)

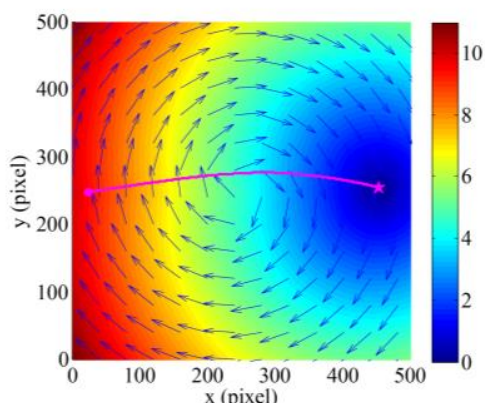

(b)

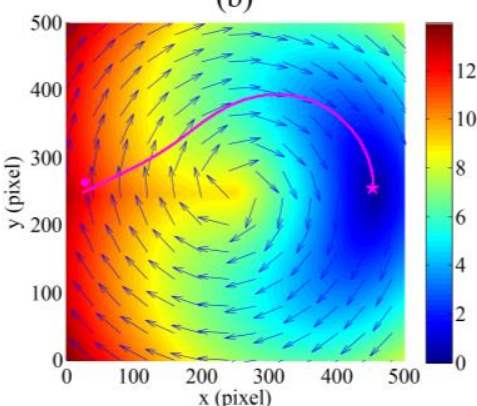

(e)

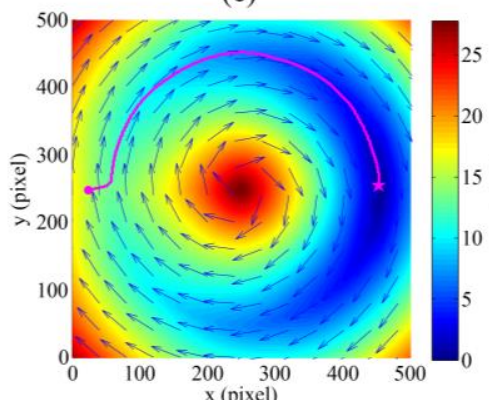

(h)

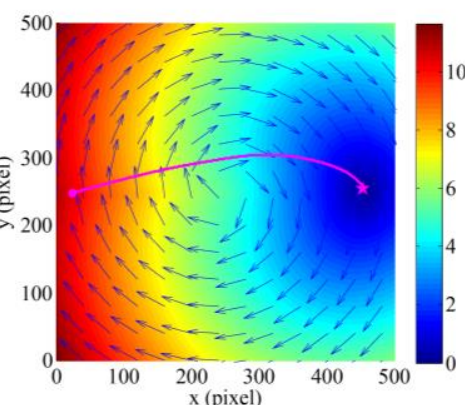

(c)

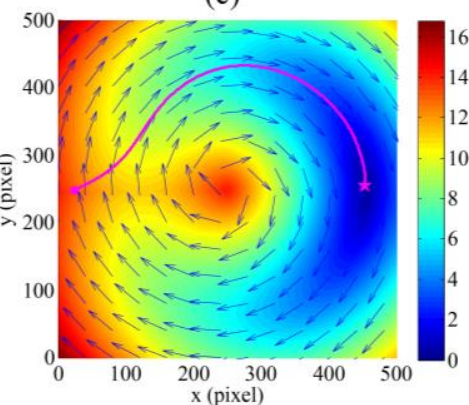

(f)

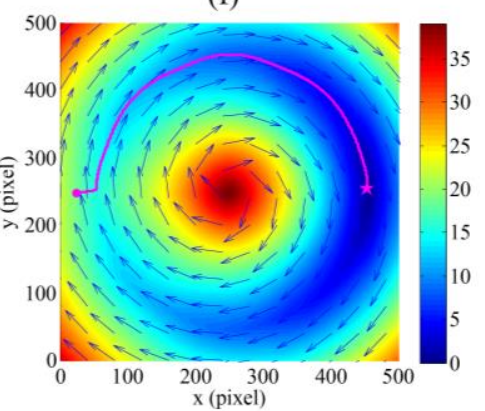

(i)

Fig. 8 Generated trajectories with different $A R$. (a)-(i) are the generated paths depicted in corresponding potential maps when $A R$ equals to $1,0.8,0.6,0.4,0.2,0.08,0.04,0.02$ and 0.01 . The environment framework of a vector field with a single 'gyre' shape (represented by blue arrows) centred in the middle. The start and goal points are marked as the shaded magenta circle and star, respectively. The coloured bars at the right-hand side depict the range of potential values with colour from blue to red. 


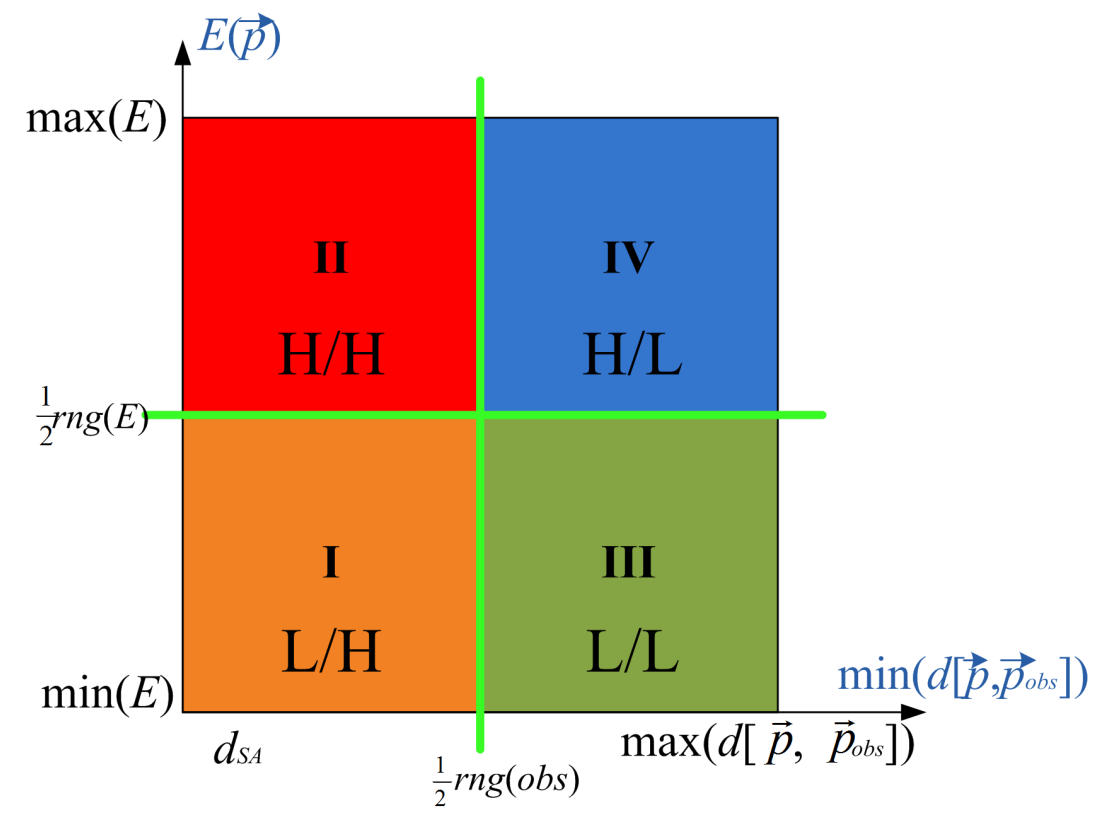

Fig. 9 4-regime risk strategy scheme. 


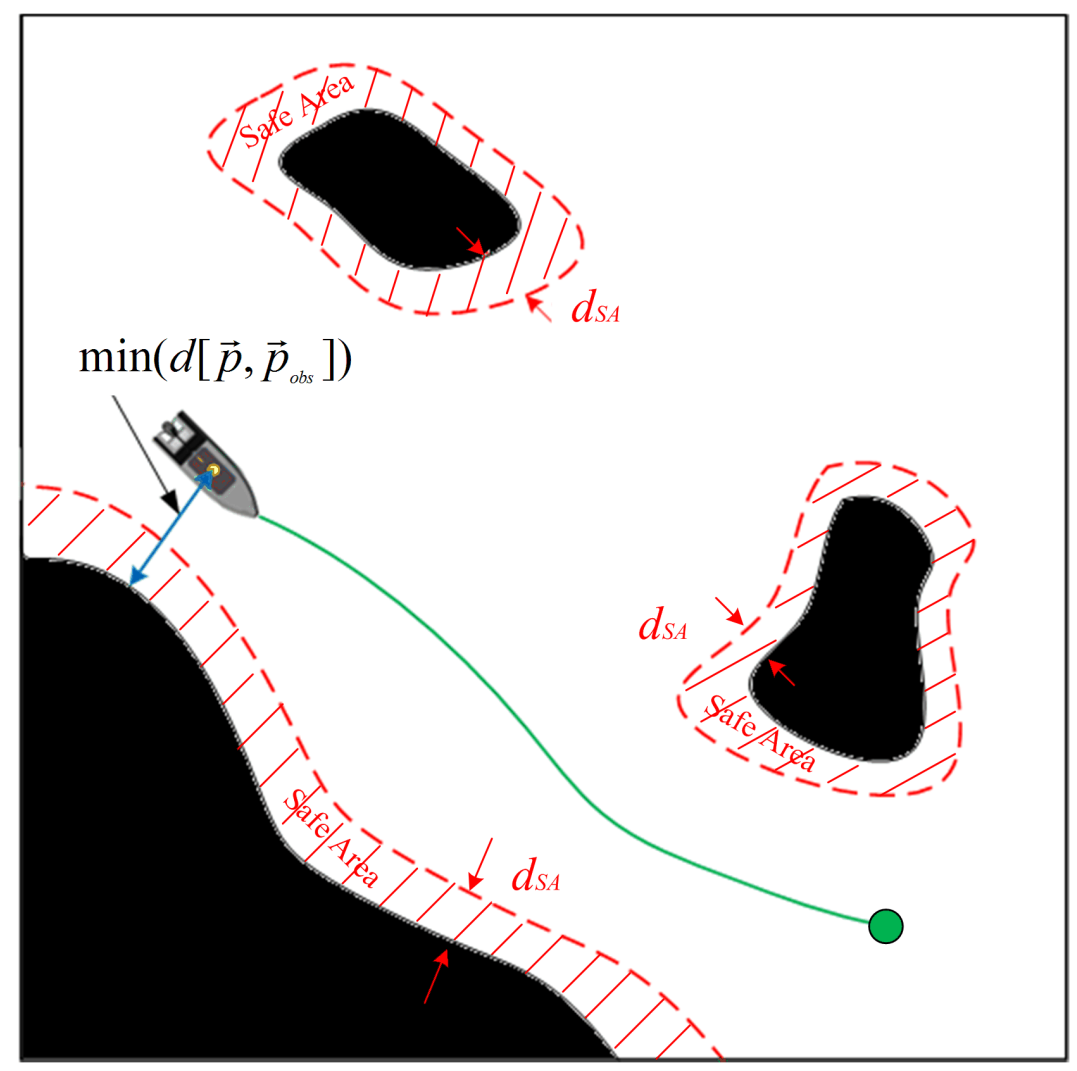

Fig. 10 Illustrations of obstacle related definitions. 


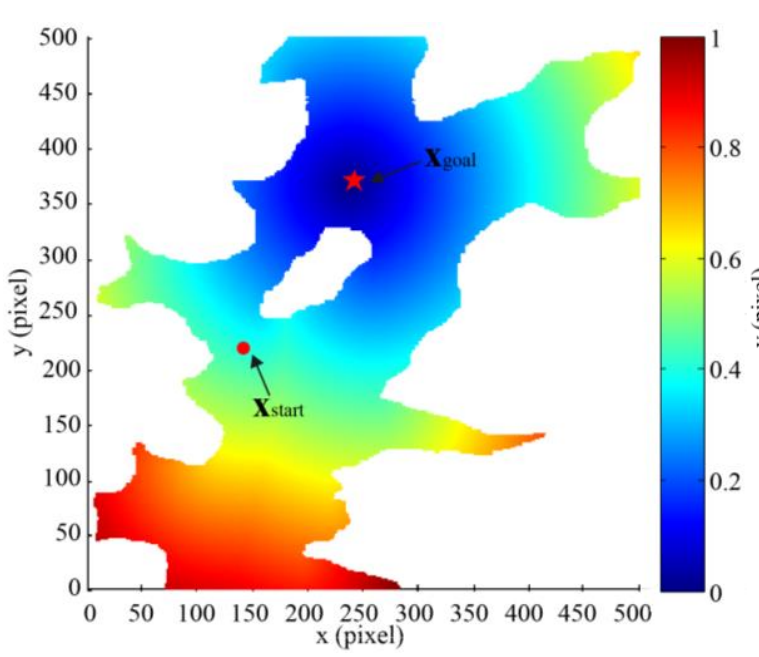

(a)

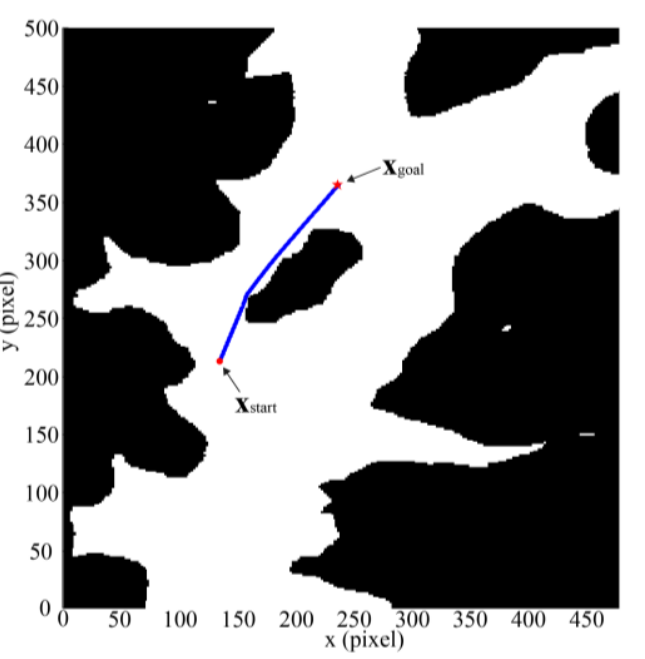

(b)

Fig. 11 Path planning results using the conventional FM method in an environment with double-gyre flow. (a) The generated potential field. (b) The generated trajectory, with computational time of $0.13 \mathrm{~s}$. The start and goal points are represented in shaded circle and star. 


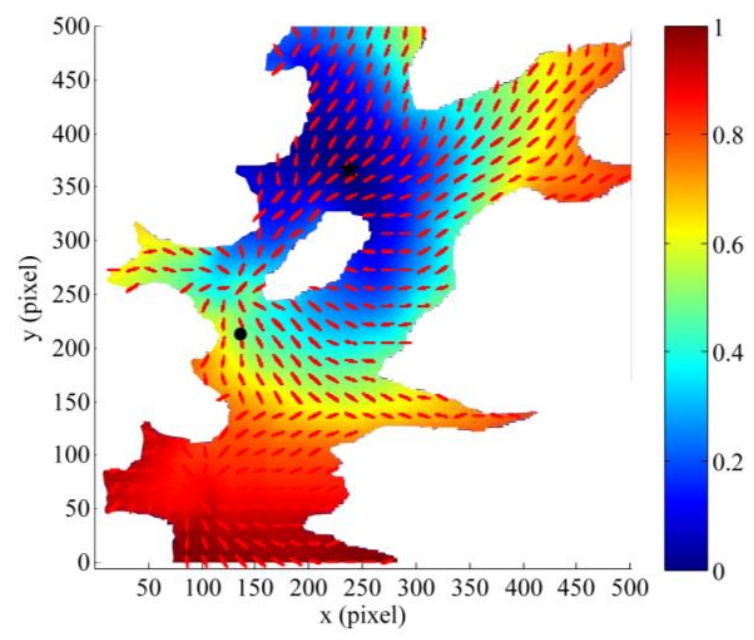

(a)

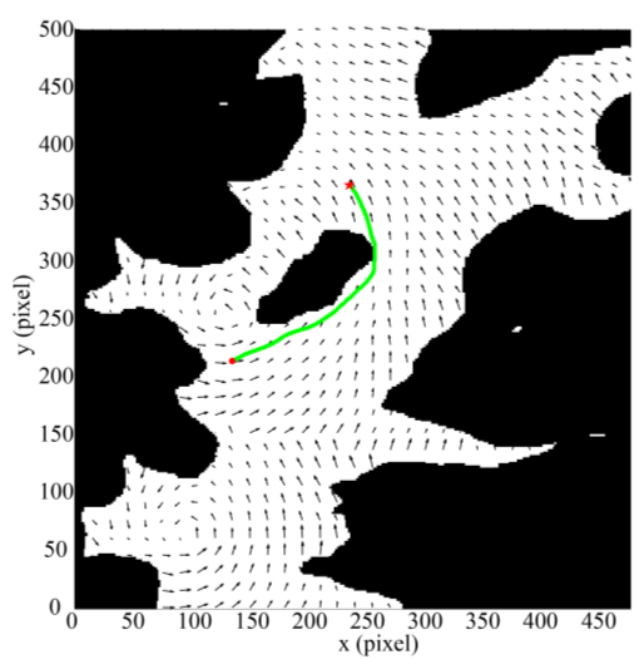

(b)

Fig. 12 Path planning results using the MFM method in the same environment with double-gyre flow as in Fig.11. (a) The generated potential field. (b) The generated trajectory, with computational time of 0.85 s. Tensor field is shown in shaded red ellipses. The start and goal points are represented in shaded circle and star. 


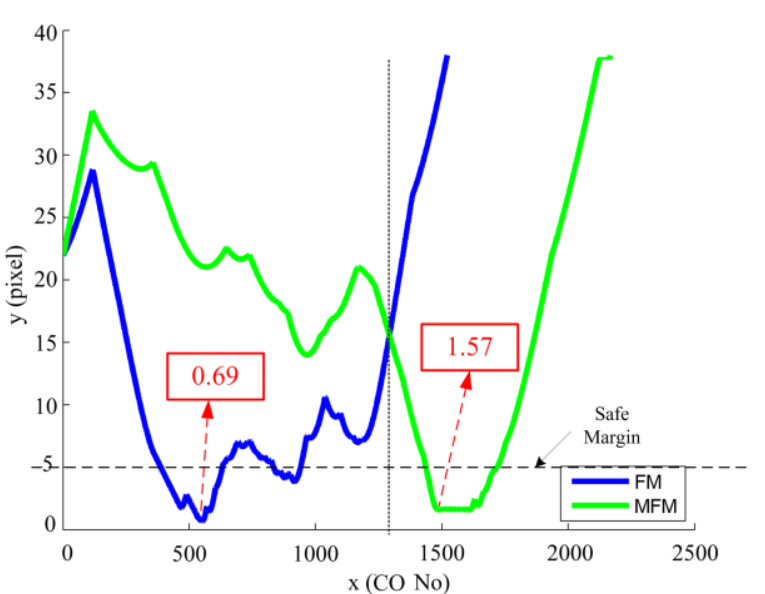

(a)

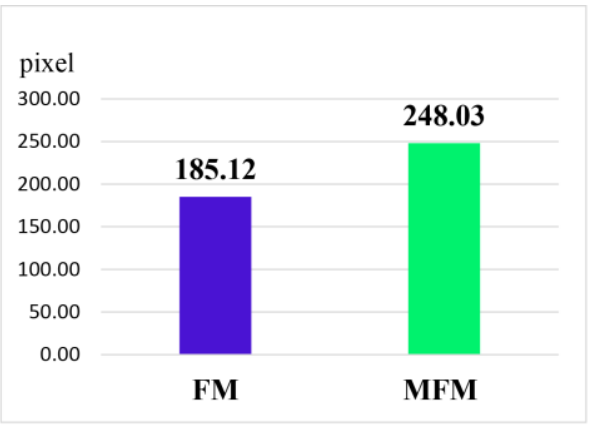

(b)

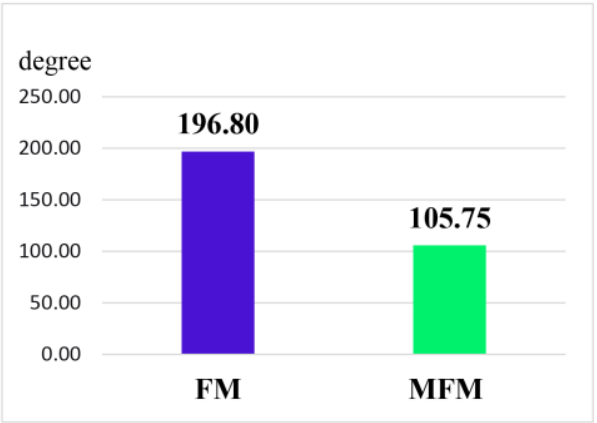

(c)

Fig. 13 Path performance comparisons between FM and MFM methods. (a) Comparison of the minimum distances to the obstacle. (b) Comparison of the total distance cost. (c) Comparison of the total energy cost. The blue results are the results of the FM method and the green ones represent the MFM results. 


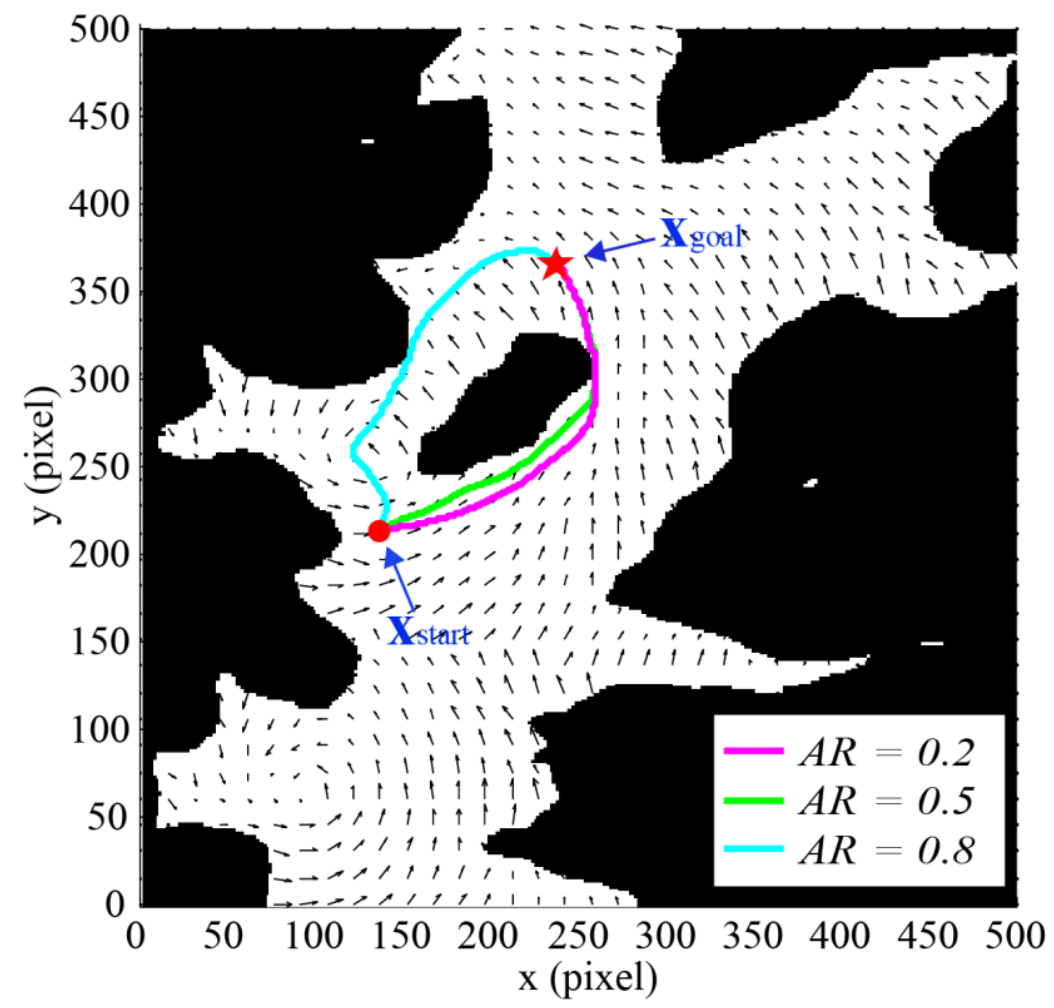

Fig. 14 Generated paths when using the MFM path planning method with the same values of field weighting $\beta_{1}=\beta_{2}=0.5$, but different values of $A R$. The magenta, green and blue colours represent the path when $A R$ equals $0.2,0.5$ and 0.8 , respectively. The computational time of each tests are $0.83 \mathrm{~s}, 0.82 \mathrm{~s}$ and 0.84 s correspondingly. 


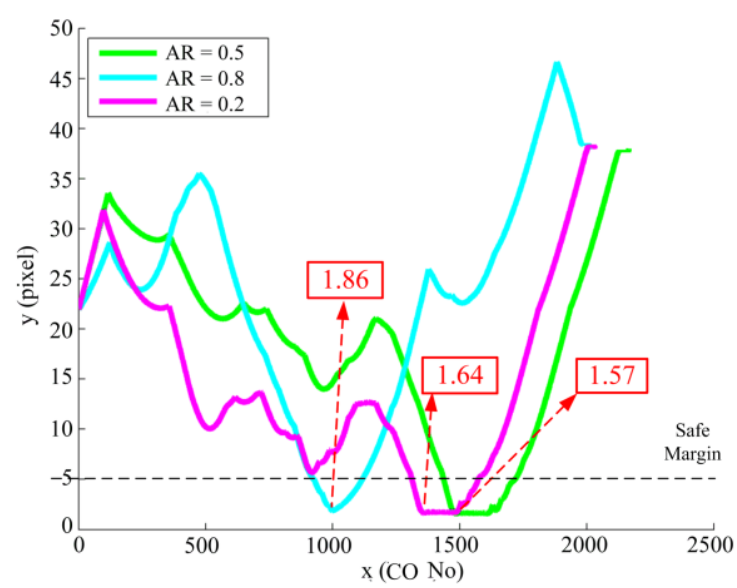

(a)

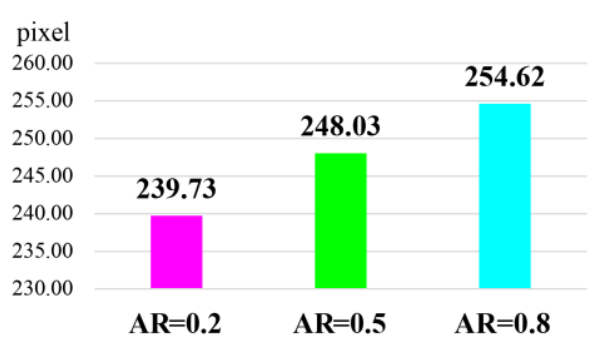

(b)

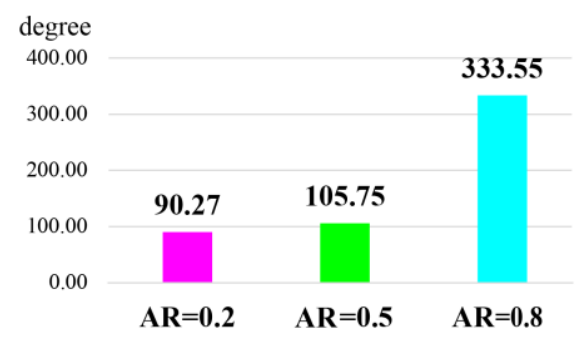

(c)

Fig. 15 Performance evaluations when using the MFM path planning method with the same values of field weighting $\beta_{1}=\beta_{2}=0.5$, but different values of $A R$. (a) Comparison of the minimum distances to obstacle. (b) Comparison of the total distance cost. (c) Comparison of the total energy cost. The magenta, green and blue colour represents the results when $A R$ equals $0.2,0.5$ and 0.8 , respectively. 


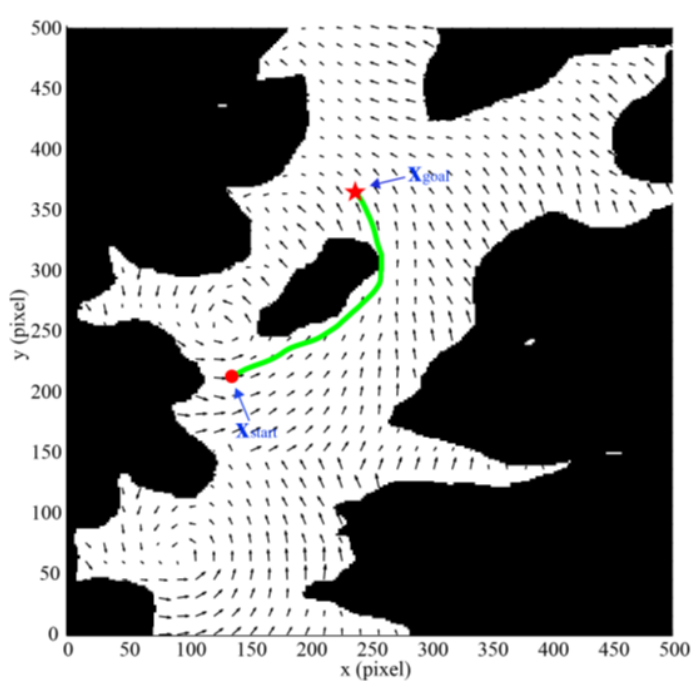

(a)

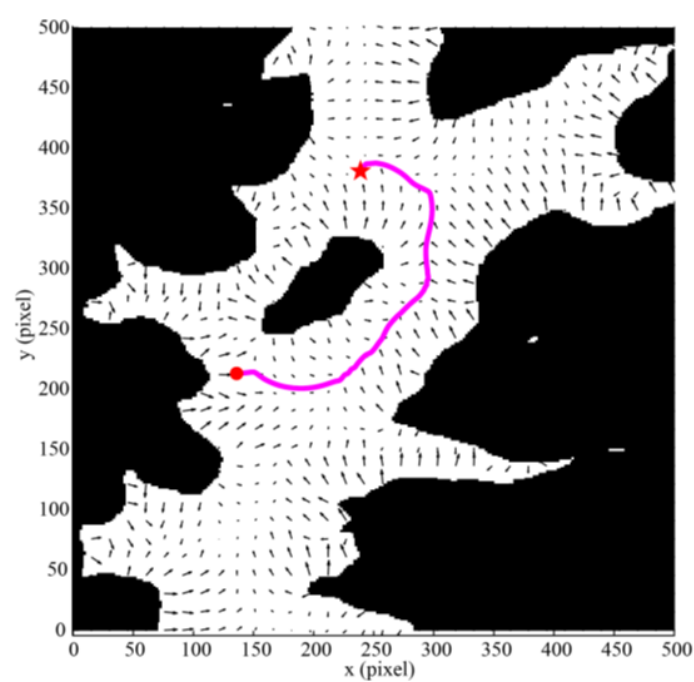

(b)

Fig. 16 Generated trajectories by using the MFM method with the same value of $A R(0.2)$, but different field weightings. (a) The green line is the path when $\beta_{1}=\beta_{2}=0.5$; (b) the magenta line is the path when $\beta_{1}=0.2$ and $\beta_{2}=0.8$. The computational time of each tests are $0.83 \mathrm{~s}$ and $0.85 \mathrm{~s}$ correspondingly. 


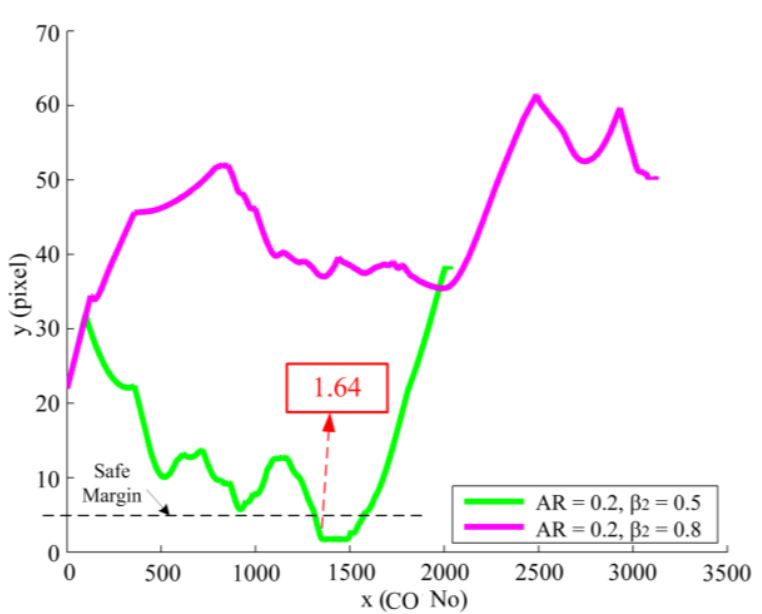

(a)

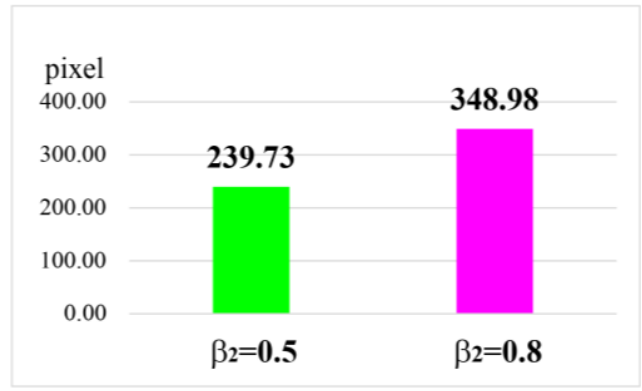

(b)

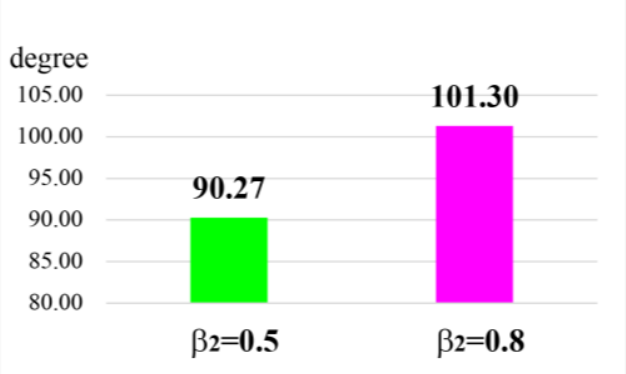

(c)

Fig. 17 Performance comparisons of the generated paths when using the MFM path planning method with the same value of $A R$, but different values of field weightings. (a) Comparison of the minimum distance to obstacles. (b) Comparison of the total distance cost. (c) Comparison of the total energy cost. The green and magenta colour represent the results when $\beta_{1}=\beta_{2}=0.5$ and $\beta_{1}=0.2, \beta_{2}=0.8$, respectively. 

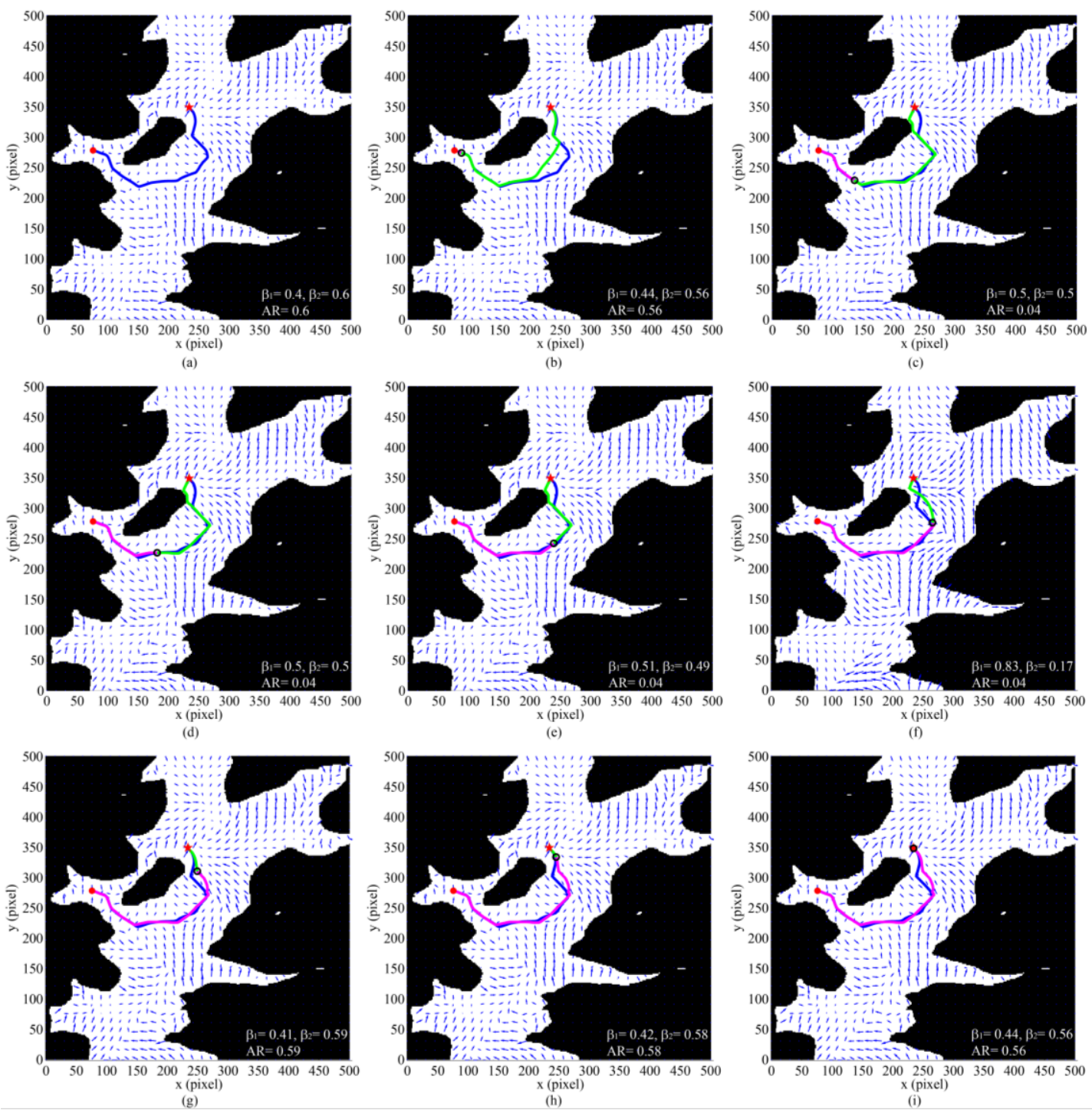

Fig. 18 Dynamic path planning results with in-variant surface currents. (a) to (f) show the generated paths at time step (t) equals to $1,2,7,11,16,19,22,24$ and 25 , with step size equals to $320 \mathrm{~m}$. The corresponding synthetic vector field is represented as blue arrows in each figure. The blue line is the generated path when USV at the start point, namely $\mathrm{t}=1$. The green line represents the updated paths at different time steps and the magenta line shows the tracking trajectory of the USV. The average computational time of each time step is around $0.91 \mathrm{~s}$. 


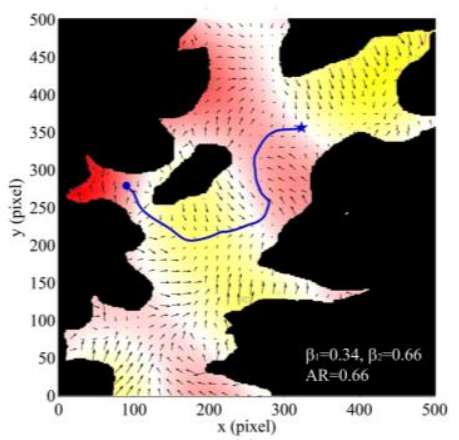

(a)

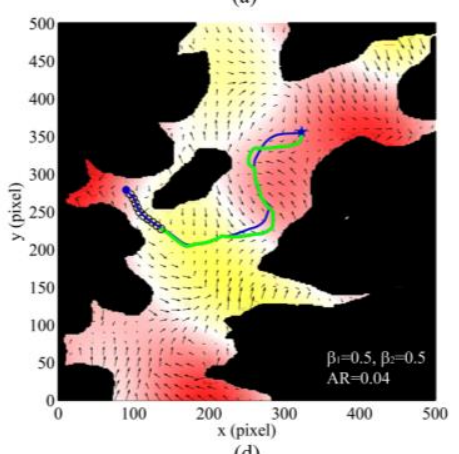

(d)

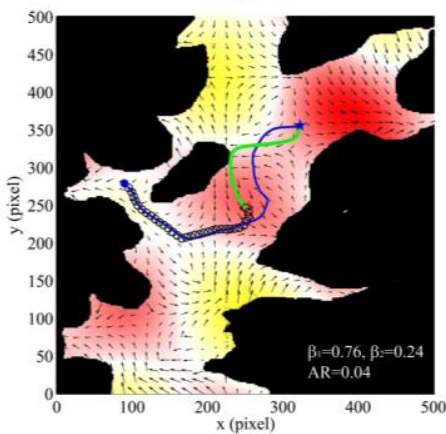

(g)

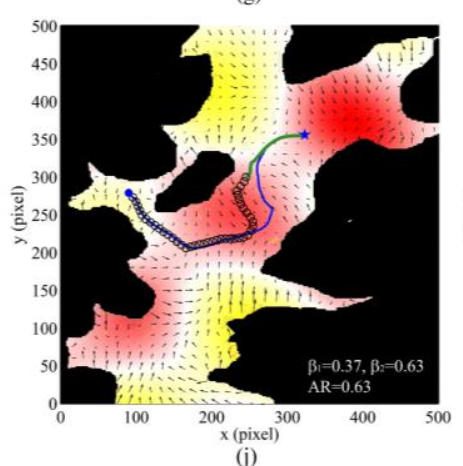

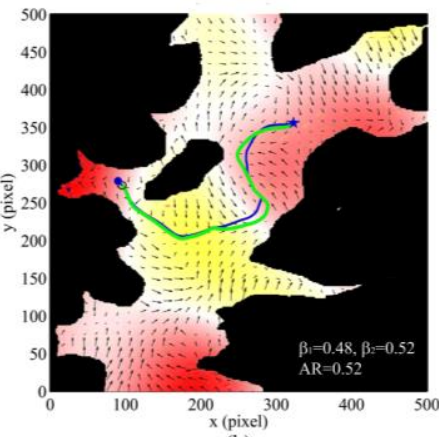

(b)

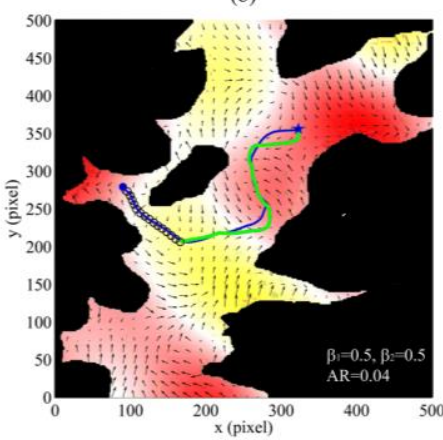

(e)

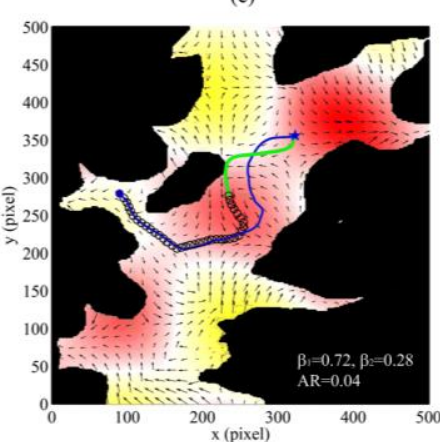

(h)

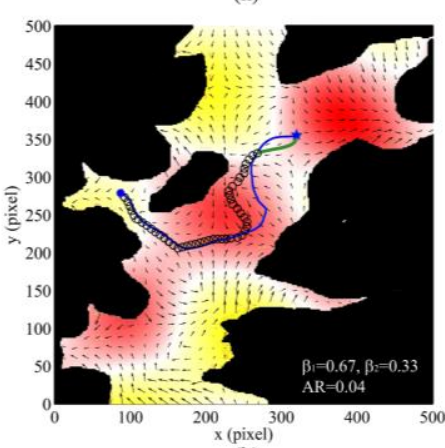

(k)

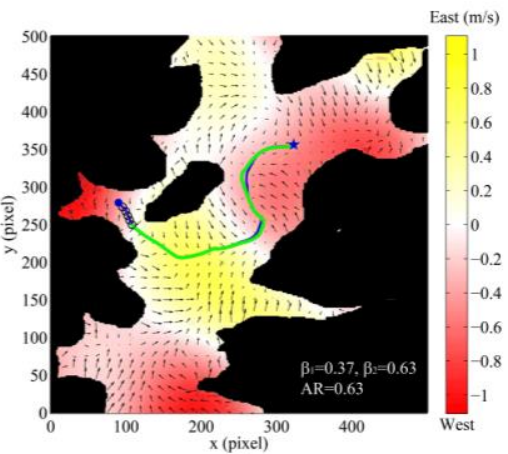

(c)
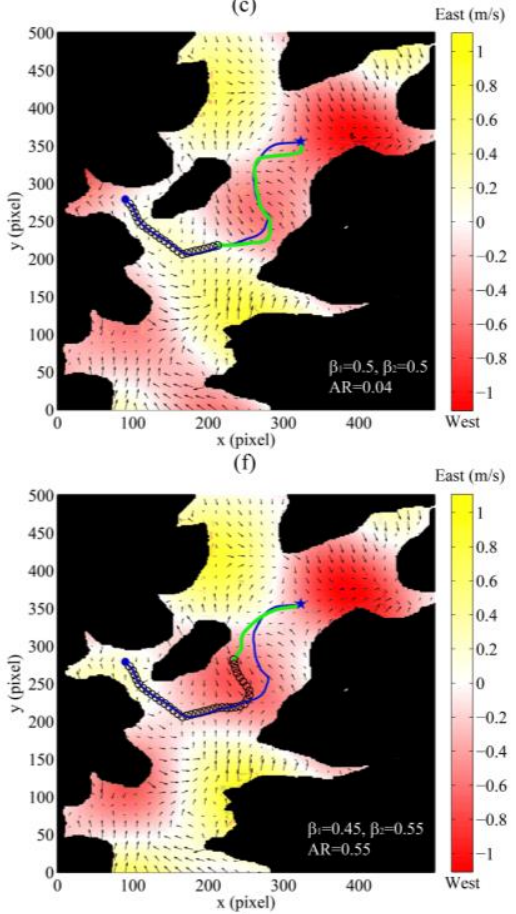

(i)

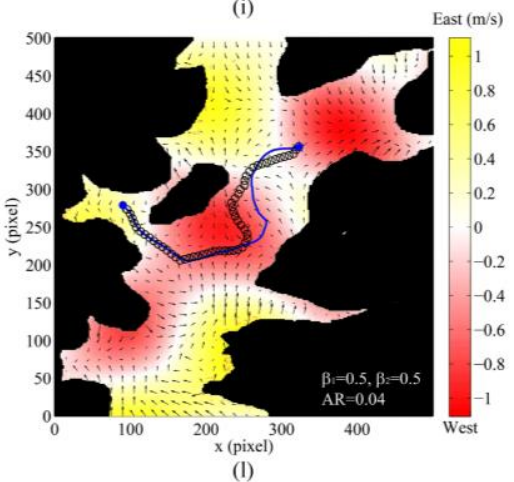

Fig. 19 Dynamic path planning results with variant surface currents. (a) to (1) show the generated paths when the time step equals to $1,2,6,11,16,24,34,39,40,43,49$ and 59 (step size $=320 \mathrm{~m}$ ). The corresponding synthetic vector field is represented as black arrows. The colour varying from red to yellow represents the surface currents directions (from the east to west) and magnitudes. The blue line is the generated path when USV at the start point, namely $t=1$. The green line represents the updated paths at different time steps and the black circle shows the tracking trajectory of the USV. The average computational time of each time step is around $0.92 \mathrm{~s}$. 
TABLE I. FIELD WEIGHTINGS IN DIFFERENT REGIME

\begin{tabular}{cc}
\hline Regime & Weightings equation \\
\hline$R_{\beta_{1}, \beta_{2}}^{1} R_{\beta_{1}, \beta_{2}}^{2}$ & $\beta_{2}=\min \left(d\left[\vec{p}, \vec{p}_{o b s}\right]\right) / r n g(o b s) ; \beta_{1}=1-\beta_{2}$ \\
$R_{\beta_{1}, \beta_{2}}^{3}$ & $\beta_{1}=\beta_{2}=0.5$ \\
$R^{4}{ }_{\beta_{1}, \beta_{2}}$ & $\beta_{1}=E(\vec{p}) / r n g(E) ; \beta_{2}=1-\beta_{1}$ \\
\hline
\end{tabular}

